\title{
Label-Free Quantitative Phase Imaging Reveals Spatial Heterogeneity of Extracellular Vesicles in Select Colon Disorders
}

\author{
Łukasz Zadka, ${ }^{*}$ Igor Buzalewicz, ${ }^{\dagger}$ Agnieszka Ulatowska-Jarża, ${ }^{\dagger}$ Agnieszka Rusak, ${ }^{*}$ Maria Kochel, ${ }^{\dagger}$ Ireneusz Ceremuga, ${ }^{\S}$ and \\ Piotr Dzięgiel*
}

From the Department of Histology and Embryology, * Department of Human Morphology and Embryology, and the Department of Medical Biochemistry, Wroclaw Medical University, Wroclaw; the Bio-Optics Group,$^{\dagger}$ Department of Biomedical Engineering, Faculty of Fundamental Problems of Technology, Wroclaw University of Science and Technology, Wroclaw; and The Institute of Geological Sciences, ${ }^{\ddagger}$ University of Wroctaw, Wroclaw, Poland

\author{
Accepted for publication \\ August 3, 2021. \\ Address correspondence to \\ Łukasz Zadka, M.D., Depart- \\ ment of Histology and Embry- \\ ology, Department of Human \\ Morphology and Embryology, \\ Chałubińskiego 6a, 50-368 \\ Wrocław, Poland. E-mail: \\ lukasz.zadka@student.umed. \\ wroc.pl or ceadr7@gmail.com.
}

\begin{abstract}
Three-dimensional (3D) imaging and quantitative analysis of extracellular vesicles (EVs) remain largely unexplored, mainly because of limitations in detection techniques. In this study, EVs from patients diagnosed with colorectal cancer (CRC) and ulcerative colitis were examined. To investigate the spatial heterogeneity and 3D refractive index (RI) distribution of single EVs, a label-free digital holographic tomography technique was used at a submicrometer spatial resolution. The presented image-processing algorithms were used in quantitative analysis with digital staining and 3D visualization, the determination of the EV size distribution and extraction of fractions with different RIs. Reconstructed 3D RI distributions revealed variations in the spatial heterogeneity of EVs related to tissue specificity, such as CRC, normal colonic mucosa, and ulcerative colitis, as well as the isolation procedures used. The RI values of EVs isolated from solid tissues of frozen CRC samples were also dependent on the tumor grade and cancer cell proliferation. The simultaneous examination of cell culture models confirmed the association of the RI of EVs with the tumor grade. 3D-RI data analysis generates new perspectives with the optical, contact-free, label-free examination of the individual EVs. Depending on the specific tissue and isolation method, EVs exhibit significant spatial heterogeneity. The optical parameters of single EVs enabled their classification into two unique subgroups with different RI values. (Am J Pathol 2021, 191: 2147-2171; https://doi.org/10.1016/j.ajpath.2021.08.005)
\end{abstract}

Exocytosis, a widespread form of intercellular communication, enables the exchange of biological content through the release of numerous extracellular vesicles $(\mathrm{EVs})$ by the cell into the extracellular environment. The unique properties and biological functions of EVs highlight their potential important biological role in the pathogenesis of many diseases. ${ }^{1}$ The diversity of EVs and the contents of their cargo are associated with the molecular complexity of cancer and show tissue specificity, which is important in the formation of distant metastases ${ }^{2}$ and disease progression. ${ }^{3}$ In addition, EVs can also act as an efficient tool in anticancer therapy, serving as a potent drug delivery system that easily overcomes the natural barriers of individual tissue compartments. ${ }^{4}$ The ability of EVs to interact with immune cells allows their membrane components to be used in nanomedicine to develop nanoparticles that regulate inflammation. ${ }^{5}$ EVs are also used in the field of regenerative medicine, ${ }^{6,7}$ and their diagnostic and predictive potential allows the use of these nanostructures as novel biomarkers for many different diseases. 8,9

However, the growing research interest in using EVs as a useful tool in clinical practice and medical research is not without limitations. There is still no established consensus on the classification of EVs, which consist of extremely heterogeneous subpopulations with different biogenesis, antigenicity, molecular features, overall biological activity,

\footnotetext{
Supported by National Science Centre (NCN) and PRELUDIUM Program grant UMO-2017/27/N/NZ5/02020 (Ł.Z.).

Disclosures: None declared.
} 
and morphometric parameters, such as size, density, and shape. ${ }^{10}$ The considerable differences in EVs contribute to the different classifications of these vesicles, whose characterization as the appropriate subtype is most often based on their diameter. ${ }^{11}$ Arbitrary classification methods allow EVs to generally be categorized as small vesicles with a maximum diameter of $200 \mathrm{~nm}$ (small EVs) and large vesicles, which exceed this size range [large EVs $(\mathrm{lEVs}){ }^{12}$

Because of the nanometer size range of some EVs, their detection remains difficult, and their visualization requires the use of, among other techniques, electron microscopy techniques, such as transmission electron microscopy or scanning electron microscopy (SEM), which require invasive procedures that may change the physicochemical properties of the sample. The proper preparation and measurement of these techniques are also extremely timeconsuming. ${ }^{13}$ These factors hinder the visualization of EVs, and extended microscope operating time and use of human resources increase the cost of these analyses.

Currently, assessing the heterogeneity of EVs poses a great methodological challenge, and optical techniques that rely on contactless and nondestructive imaging have become increasingly important novel analytical methods. However, most of these methods use external reagents and fluorescence markers to detect individual EVs. ${ }^{14}$ Digital holographic tomography (DHT) is a new label-free technique that enables the vital assessment of cells visualized in three-dimensional (3D) images based on the determined 3D distribution of the refractive index (RI) of the tested objects and measured physical and biological properties. DHT is based on limited-angle holographic tomography,${ }^{15}$ in which the scattered field from the sample is collected by an objective lens and in the camera plane interfering with the reference beam. Consequently, it is possible to register a series of digital holograms for various incident angles of the illumination beam. From each digital hologram, it is possible to reconstruct the two-dimensional (2D)-RI tomogram representing a single slice of the 3D-RI distribution (3D-RI tomogram) using the principle of optical diffraction tomography. ${ }^{16,17}$ The theoretical lateral and axial resolution of DHT systems is 124 and $397 \mathrm{~nm}$, respectively, ${ }^{16,18}$ and provides morphologic and biochemical information with submicrometer ${ }^{19-22}$ or even subnanometer ${ }^{23}$ resolution, which could make them suitable for the examination of single EVs below the resolution limit of classical optical microscopy. Recently, it was reported that DHT has the potential to become an important diagnostic tool for the examination of different biological samples. ${ }^{1,21,22}$ The obtained DHT results have a quality comparable to that of electron microscopy techniques, ${ }^{24}$ which emphasizes the theoretical potential of this method in the vital imaging of nanostructures, such as EVs. The aim of this study was to investigate the potential of the DHT method in imaging and the characterization of the 3D-RI distribution and morphology of single EVs to verify their heterogeneity and examine the influence of sample preparation procedures on the reconstructed RI data. This is the first attempt to determine the 3D-RI distribution of single EVs, as until now, only indirect RI measurements with nanoparticle tracking analysis (NTA) have been reported. ${ }^{25}$

Moreover, the DHT technique was also used to verify the potential use of RI data for the differentiation of selected gastrointestinal disorders based on the differences in the spatial heterogeneity and 3D-RI of EVs isolated from different tissues. Chronic inflammation of the colonic mucosa and molecular-structural changes that occur during colitis are key factors in the development of colorectal cancer (CRC). ${ }^{26}$ This cancer is predominantly linked to intestinal inflammation, which is one of the main pathogenetic factors that leads to its development, ${ }^{27}$ this makes it relatively common for precancerous lesions to develop in patients with inflammatory bowel diseases, such as ulcerative colitis (UC). ${ }^{26}$ Currently, unmet needs in the diagnosis of CRC mainly involve the identification of new biomarkers with diagnostic and prognostic significance; the important role that EVs appear to play in the pathogenesis of this common cancer suggests their potential usefulness in the early screening of CRC. ${ }^{28,29}$

In this study, the heterogeneity of single EVs was evaluated using DHT analysis of exosomal pellets isolated with different methodological approaches from patients diagnosed with CRC and UC. Human plasma microsamples and solid tissue isolates were used for vesicle preparation. With DHT, the relationship of imaged EV features and the impact of various isolation approaches were examined. Ultracentrifuged CRC isolates were compared with the corresponding normal mucosa of the large intestine. SEM micrographs and immunoblotting were performed on EV pellets using commercially available reagents. The optical parameters of single EVs were compared with the wellknown, routine histopathologic features with predictive value, such as tumor grade and cell proliferation. ${ }^{30,31}$ Complementary analyses were performed with selected in vitro cell culture models.

\section{Materials and Methods}

\section{Patients and Samples}

The tissue samples used in this study were collected during endoscopic procedures of the lower gastrointestinal tract in patients hospitalized at the Gastroenterology Department of the 4th Military Hospital (Wrocław, Poland) in January and February 2020. Fresh human plasma samples were prepared from venous blood collected before colonoscopy from patients with an initial diagnosis of CRC and suspected inflammatory bowel disease. During endoscopy, solid tissue samples from lesions were also collected for histopathologic analysis as a part of routine clinical diagnosis. Additional solid tissue material was stored for research purposes in a fresh $4 \%$ paraformaldehyde solution to generate 
Table 1 Clinicopathologic Characteristic of Patients Included in This Study for DHT Analysis $(n=15)$

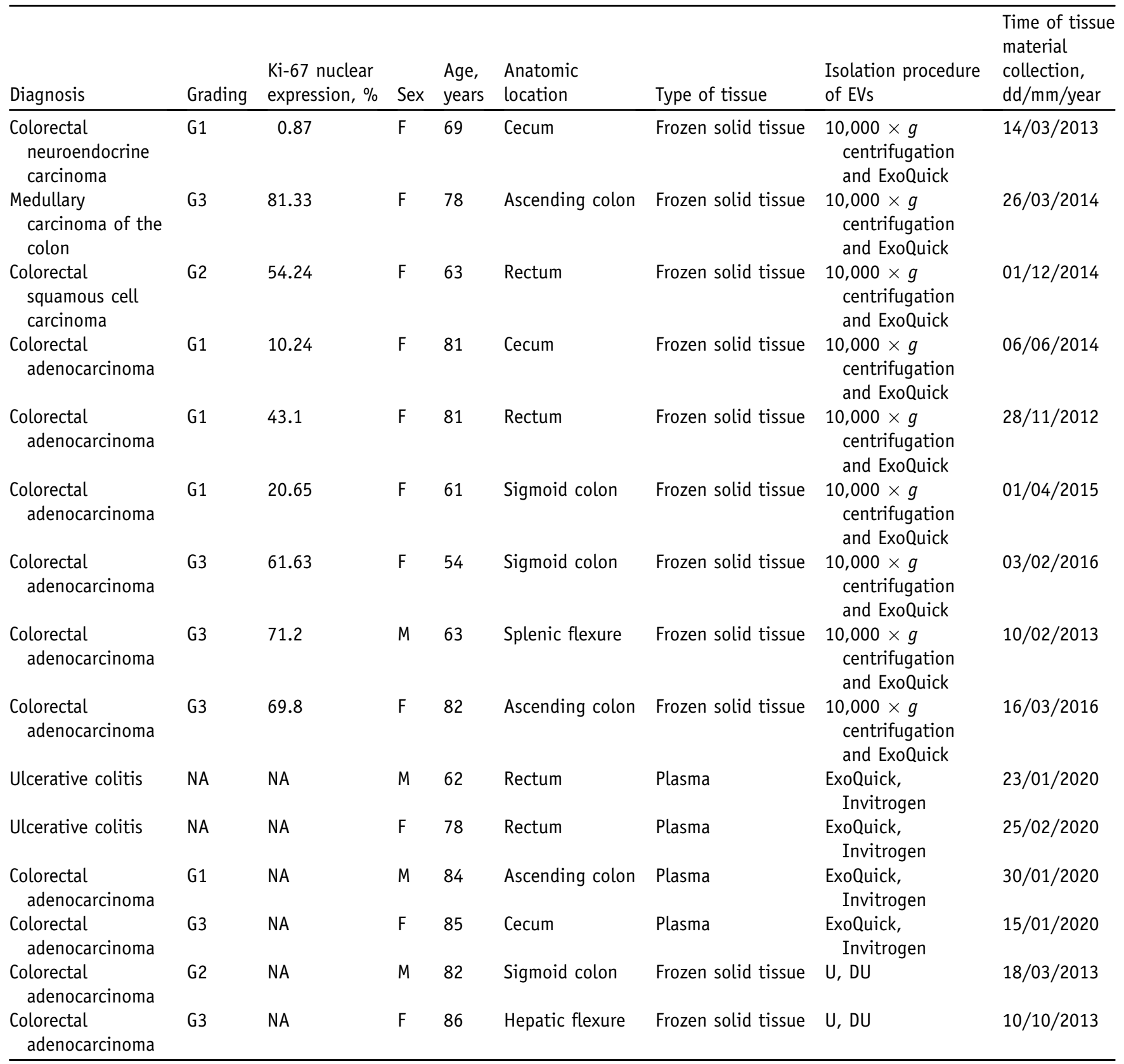

F, female; M, male; dd, date; DHT, digital holotomography; DU, differential ulctracentrifugation; EV, extracellular vesicle; G, grade; mm, month; NA, not applicable; $U$, ultracentrifugation.

micrographs of ultrathin biopsies and for further molecular studies. On the basis of the histopathologic diagnosis, four patients qualified for the study: two with a diagnosis of CRC and two with a diagnosis of UC. Moreover, the study included archived tissue samples stored at $-80^{\circ} \mathrm{C}$ from 11 patients with primary CRC. Two of these patients had primary adenocarcinomas, from which the isolated EVs were also compared with vesicles obtained from paired cancer-associated normal colonic mucosa. The archival material was collected during colectomy procedures performed at the Surgery Clinic of the 4th Military Hospital in
2012 to 2016. The surgical margins assessed in this study, defined as normal controls $(n=2)$, were additionally verified for the presence of cancer cells by standard hematoxylin and eosin staining; routinely stained histologic slides excluded any neoplastic infiltrates. Each of the patients included in the study provided written consent for the appropriate molecular tests. The study protocol was approved by the Bioethics Committee at Wroclaw Medical University (Wroclaw, Poland; protocol number 609/2021). The clinicopathologic data of patients included in this study are shown in Table 1. 


\section{Human Plasma Preparation}

Plasma isolation was performed on venous blood collected (volume of $12 \mathrm{~mL}$ ) into $\mathrm{K}_{2}$ EDTA tubes from four patients undergoing colonoscopy. Venous blood was collected at the gastroenterology ward and then transported to the Department of Histology and Embryology at Wroclaw Medical University within 40 minutes. The blood samples were pipetted into sterile $1.5-\mathrm{mL}$ Eppendorf tubes to obtain plasma with centrifugation at $2000 \times g$ for 20 minutes at $4^{\circ} \mathrm{C}$ (Sorvall Legend X1R Centrifuge; Thermo Fisher Scientific, Waltham, MA). Next, the obtained plasma was separated from the remaining blood morphotic elements and placed in new Eppendorf tubes.

\section{Extraction of EVs from Solid Tissue Isolates}

Frozen tissue was obtained during colectomy in patients diagnosed with CRC. Samples were gently thawed on ice and weighed with an electronic balance, and $100 \mathrm{mg}$ per sample was then placed in fresh Eppendorf tubes. EV isolation was performed according to previously published protocols. ${ }^{28,32}$

\section{Sample Purification Procedure}

The isolated plasma was stored at $-20^{\circ} \mathrm{C}$ until further analyses. After gently thawing the samples on ice, differential plasma centrifugation was performed in the sequence as follows: $300 \times g$ for 5 minutes at $4^{\circ} \mathrm{C}, 2000 \times g$ for 10 minutes at $4^{\circ} \mathrm{C}$, and $10,000 \times g$ for 30 minutes at $4^{\circ} \mathrm{C}$. The pellets were discarded each time, and the obtained supernatants were placed in new Eppendorf tubes. The same parameters were applied to purify the supernatant of EV isolates obtained from solid tissues.

\section{Precipitation of EVs from Human Plasma Microsamples}

Purified plasma samples ( $250 \mu \mathrm{L}$ each) were used to obtain EV pellets. Commercially available reagents from various manufacturers were used to precipitate the pellets: ExoQuick Exosome Precipitation Solution for Serum, Plasma and Tumor Ascites Fluid $(20 \mathrm{~mL}$; catalog number 322EXOQ20A-1; System Biosciences, Palo Alto, CA) and an Invitrogen Total Exosome Isolation Kit (TEIK; catalog number 4484450; Thermo Fisher Scientific). The EV isolation procedure was performed according to the provided protocols. For the Invitrogen TEIK, the protocol without proteinase treatment was applied by diluting $250 \mu \mathrm{L}$ of human plasma in $125 \mu \mathrm{L}$ of Dulbecco's phosphate-buffered saline (PBS; $1000 \mathrm{~mL}$; catalog number 14190136; Gibco, Thermo Fisher Scientific) and vortexing. Then, $75 \mu \mathrm{L}$ of TEIK was added. After vortexing, $450 \mu \mathrm{L}$ of each sample was incubated at room temperature for 10 minutes, and then centrifuged at $10,000 \times g$ for 5 minutes at room temperature. The obtained supernatant was discarded, and the EV pellet was suspended in $50 \mu \mathrm{L}$ Dulbecco's PBS and then stored at $-20^{\circ} \mathrm{C}$. For the ExoQuick method, $63 \mu \mathrm{L}$ of the reagent was added to $250 \mu \mathrm{L}$ of plasma. Next, the samples were vortexed and incubated at $4^{\circ} \mathrm{C}$ for 1 hour. Then, the samples were centrifuged at $1500 \times g$ for 30 minutes at $4^{\circ} \mathrm{C}$. After centrifugation, the supernatant was removed, and the obtained exosomal pellet was suspended in $50 \mu \mathrm{L}$ of Dulbecco's PBS and stored at $-20^{\circ} \mathrm{C}$. All fresh samples were transported on ice to visualize the EVs with DHT imaging.

\section{Differential Ultracentrifugation on a Sucrose Cushion}

According to the protocol of Vella et al, ${ }^{32}$ a sucrose gradient (Sigma, St. Louis, MO) with concentrations of 205, 445, and $855 \mathrm{~g} / \mathrm{L}$ (F1, F2, and F3 fractions, respectively) was prepared in PA Ultracrimp tubes (catalog number 03987; Thermo Scientific, Waltham, MA). Supernatant $(750 \mu \mathrm{L})$ obtained from the isolation was pipetted onto the prepared gradient and subjected to ultracentrifugation on a T-890 rotor with the following centrifugation parameters: $180,000 \times g, 3$ hours, and $4^{\circ} \mathrm{C}$. Fraction F2 was then collected, and the sample contents were diluted with icecold Dulbecco's PBS. The samples were centrifuged at $100,000 \times g$ at $4^{\circ} \mathrm{C}$ for 1 hour using PA Cone-top tubes (part number 9012; Seton Scientific, Petaluma, CA) and a TFT 80.4 rotor. The obtained pellet was suspended in $50 \mu \mathrm{L}$ of inhibitor cocktail and stored at deep freezing temperatures until further analyses were performed.

\section{Ultracentrifugation}

Supernatant samples $(750 \mu \mathrm{L})$ containing the EVs obtained from solid tissue isolates were pipetted into fresh and sterile Eppendorf tubes. The samples were transported on ice within 5 minutes in a Styrofoam box to the Department of Medical Biochemistry at Wroclaw Medical University. The supernatants were pipetted into new tubes, PA Cone-top tubes, and centrifuged in a Sorvall WX Ultra 100 ultracentrifuge (Thermo Scientific) using TFT 80.4 rotor, according to the protocol of Théry et al. ${ }^{33}$ The EV pellets were suspended in $50 \mu \mathrm{L}$ of inhibitor cocktail, and then stored at $-80^{\circ} \mathrm{C}$ until further analysis.

\section{Cell Lines}

The human cell lines, HT-29 (adenocarcinoma, grade 1), CaCo-2 (adenocarcinoma, grade 2), LoVo (adenocarcinoma, Dukes' type C, grade 4, metastasis to nodule), $\mathrm{CCD}$ 18Co (normal colon epithelium), and HMEC-1 (microvessel endothelial cells), were obtained from ATCC (Manassas, VA). HT-29 cells were cultured with Mc-Coy's 5A (ATCC), LoVo cells with F12-K media (ATCC), CCD18$\mathrm{Co}$ and $\mathrm{CaCo}-2$ cells with Eagle's minimum essential medium [Lonza, Basel, Switzerland; with 1\% sodium pyruvate and $1 \%$ nonessential amino acids (all from Sigma-Aldrich, St. Louis, MO)], and HMEC-1 cells with MCDB-131 supplemented with fibroblast growth factor and hydrocortisone 
(Thermo Fischer). All media contained 1\% L-glutamine and penicillin-streptomycin solution and $10 \%$ fetal bovine serum (Sigma-Aldrich), except CaCo-2 cell medium, which contained $20 \%$ fetal bovine serum. Cell cultures were incubated at $37^{\circ} \mathrm{C} / 5 \% \mathrm{CO}_{2}$ and $95 \%$ humidity. Medium was changed twice a week, and cells were passaged at approximately $70 \%$ confluence. Cells were trypsinized with $0.25 \%$ trypsin-EDTA solution (Sigma-Aldrich), and the cell pellet was washed in PBS and stored at $-80^{\circ} \mathrm{C}$. Conditioned media samples were collected for EV pellet isolation.

\section{Immunoblotting}

Cell and exosomal pellets were lysed in radioimmunoprecipitation assay buffer, and tissue samples were homogenized in T-PER Tissue Protein Extraction Reagent with EDTA, a protease inhibitor cocktail (Heat Protease Inhibitor Cocktail $\times 100)$ and $0.5 \mathrm{mmol} / \mathrm{L}$ phenylmethanesulfonyl fluoride (Thermo Scientific). Lysates were denatured in sample buffer [ $250 \mathrm{mmol} / \mathrm{L}$ TRIS, $\mathrm{pH} 6.8,40 \%$ glycerol, $20 \%$ (v/v) $\beta$-mercaptoethanol, $0.33 \mathrm{mg} / \mathrm{mL}$ bromophenol blue, and $8 \%$ SDS] for 5 minutes at $95^{\circ} \mathrm{C}$. Total protein levels were determined with bicinchoninic acid (Pierce BCA Protein Assay Kit; Thermo Fisher Scientific) and the NanoDrop1000 (Thermo Scientific). Electrophoretic separation in $10 \%$ polyacrylamide gel in a Mini Protean 3 apparatus (Bio-Rad, Marnes-la-Coquette, France) was performed according to the Laemmli method with $20 \mu \mathrm{g}$ of protein per lane. ${ }^{34}$ Protein transfer was performed in Trisglycine buffer with $20 \%$ methanol and $0.05 \%$ SDS on polyvinylidene difluoride membranes (Immobilon; Millipore, Bedford, MA) for 1 hour at $140 \mathrm{~V}$. The membranes were blocked in 5\% milk in $0.05 \%$ Tris-buffered saline with Tween 20 (TBST; for exosome antibodies) or $4 \%$ bovine serum albumin in $0.1 \%$ TBST (for $\beta$-tubulin); and incubation with primary antibodies directed against heat shock protein 70 , CD63 (1:1000; in 5\% milk in 0.05\% TBST; EXOAB; System Biosciences), and $\beta$-tubulin (1:1000; in $0.1 \%$ bovine serum albumin in 0.1\% TBST; ab6046; Abcam, Cambridge, UK) was performed overnight at $4^{\circ} \mathrm{C}$. Next, the membranes were washed three times in $0.05 \%$ TBST and incubated with secondary antibodies conjugated with horseradish peroxidase for 1 hour at room temperature: goat anti-rabbit antibody (1:20,000; in 5\% milk in 0.05\% TBST; EXOAB) and donkey-anti-rabbit antibody (1:6000; in 0.1\% TBST; Jackson ImmunoResearch, Suffolk, UK). The detection of the chemiluminescence reaction was performed with Luminata Forte Immobilon Forte Western horseradish peroxidase substrate (Millipore) and a ChemiDoc MP System (Bio-Rad, Hercules, CA) with exposure times from 1 second to 1 minute using ImageLab software version 6.1 (Bio-Rad).

\section{Precipitation of EVs from Cell Culture Samples}

To isolate EVs, $8 \mathrm{~mL}$ of culture media was collected for each cell line. The culture medium sample from each cell line was then divided into two 4-mL samples in two clean 20 -mL conical tubes. Sample purification was performed according to the manufacturer's protocol with differential centrifugation. After pipetting the supernatant, the appropriate volume of ExoQuick reagent was added to new tubes $(1008 \mu \mathrm{L}$ per $4 \mathrm{~mL})$. After gentle shaking, all samples were allowed to be incubated at $4^{\circ} \mathrm{C}$ overnight. The next morning, the last stage of $\mathrm{EV}$ pellet precipitation was performed by centrifuging the samples at $4^{\circ} \mathrm{C}$ for 30 minutes at a speed of $1500 \times g$. The obtained exosomal pellets were used for DHT imaging and immunoblotting.

\section{Immunofluorescence}

HT-29, CaCo-2, LoVo, CCD-18Co, and HMEC-1 cells were seeded $\left(7 \times 10^{3}\right.$ per well) on EZ slides (Millipore) and cultured in appropriate medium. Cell fixation and permeabilization were performed with cold methanol (Chempur, Piekary Slaskie, Poland) for 10 minutes at $-20^{\circ} \mathrm{C}$, washed in PBS, and blocked with $3 \%$ bovine serum albumin in $0.1 \%$ TBST (1 hour, room temperature). Next, HT-29, CaCo-2, LoVo, and CCD-18Co cells were incubated with a cocktail of primary antibodies directed against pan-cytokeratin [ready to use (RTU); Dako, Santa Clara, CA; clone AE1/AE3] and CD63 (1:400; Sigma-Aldrich; SAB4301607). HMEC-1 cells were treated with monoclonal mouse anti-CD31 (RTU; Dako; clone JC70A) and human polyclonal rabbit antibodies against CD63 (1:400; Sigma-Aldrich; SAB4301607). Incubation was performed overnight at $4^{\circ} \mathrm{C}$. Next, the cells were washed in PBS and incubated with Alexa Fluor 568 conjugated antibody (dilution 1:2000; Abcam; ab175470) and anti-mouse Alexa Fluor 488 conjugated antibody (dilution 1:2000; Abcam; ab150113). Slides were covered with FluoroShield mounting medium with DAPI (Abcam; ab104139). Immunofluorescence microscopy (BX51; Olympus, Tokyo, Japan) with CellSens Standard imaging software version 1.15 (Olympus) was used for visualization.

\section{Protein Precipitation}

Total protein precipitation from conditioned culture media and from EV suspensions from tissue, cells, and conditioned media was performed on ice for 2 hours after adding the following reagents at these final concentrations: $1 \%$ sodium deoxycholate and 10\% trichloroacetic acid (Sigma-Aldrich). Then, the samples were centrifugated (3999 relative centrifugal force, 10 minutes), and the precipitates were dissolved in cold acetone $\left(-20^{\circ} \mathrm{C}\right.$; Chempur, Piekary Śląskie, Polska) and centrifuged (3999 relative centrifugal force, 10 minutes). ${ }^{35}$ After repeating this step two times, the acetone was removed, and the pellets were allowed to dry. Next, the pellets were suspended in radioimmunoprecipitation assay buffer with phenylmethanesulfonyl fluoride, EDTA, and a proteinase inhibitor cocktail, and the total protein concentration was measured with the bicinchoninic acid method as for the protein isolated from tissue or cell samples. 
B Digital staining of different EV fractions

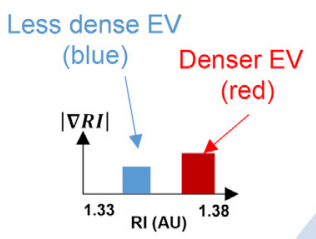

A

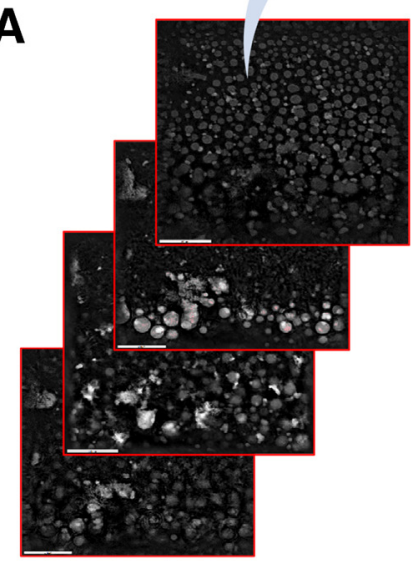

2D-RI tomograms

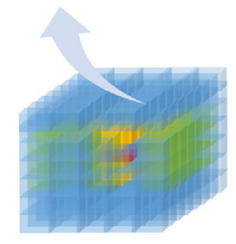

3D RI-data

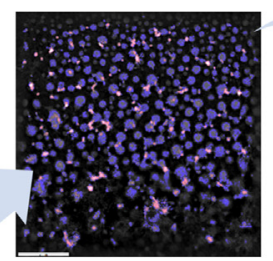

C 3D digital staining of different EV fractions and background

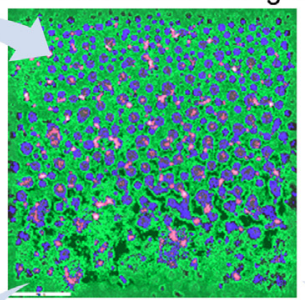

Separation of the two fractions of

EV and the background from each 2D-RI tomogram Blue Red denser EV eaction

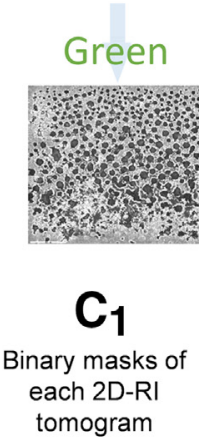
less dense EV
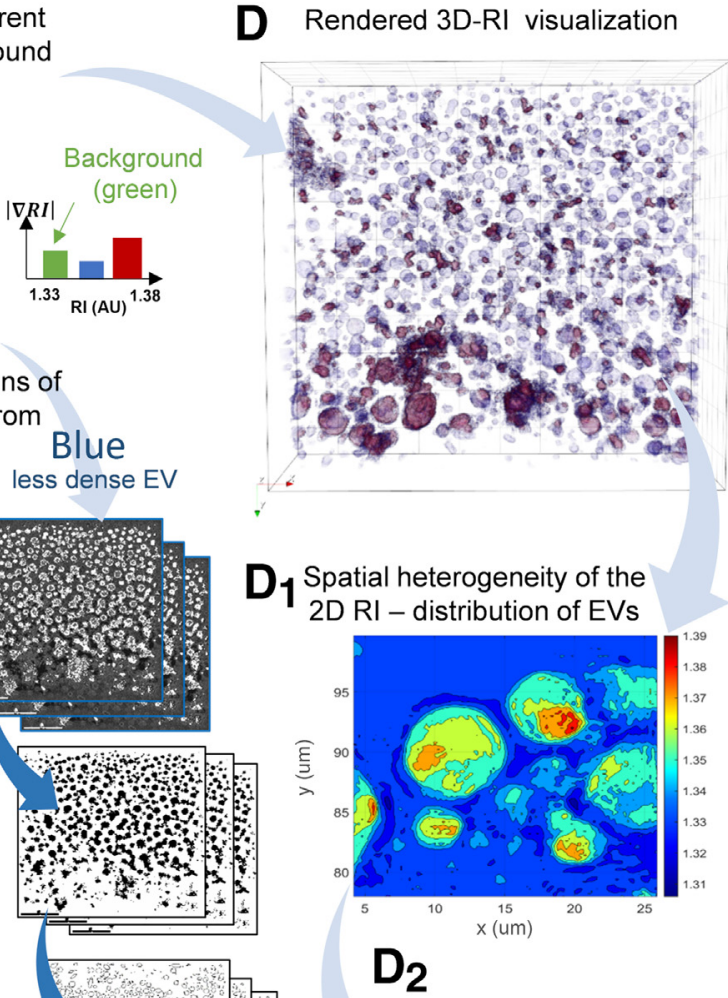

\section{$\mathrm{C}_{2}$}

Extraction of the regions of individual EV (shape, diameter)

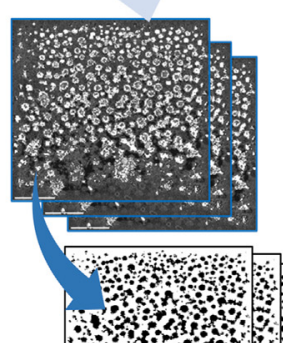

$D_{1}$ Spatial heterogeneity of the

Variation of RI values among two fractions of EVs
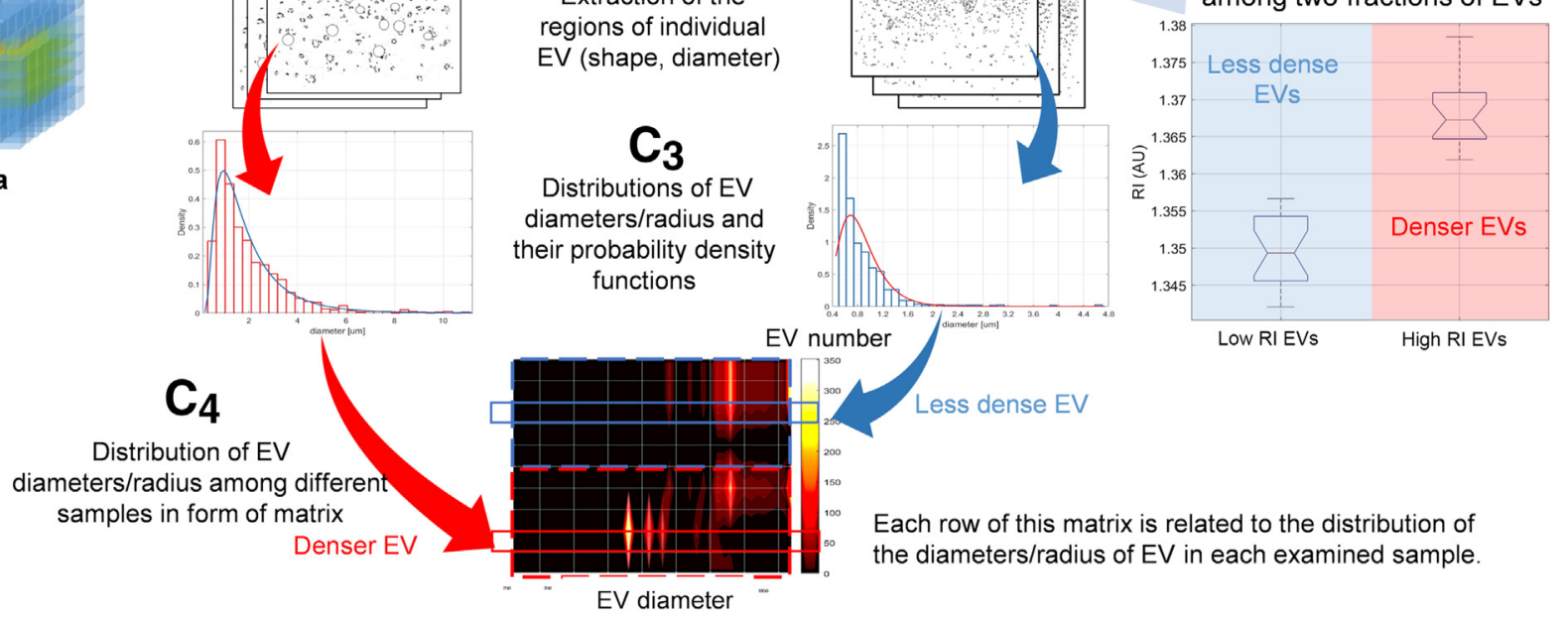

Figure 1 Workflow of extracellular vesicle (EV) examination and refractive index (RI) data processing. The reconstruction of the series of two-dimensional (2D)-RI tomograms (A), digital staining of EVs (B) and the surrounding reagent (C), rendered visualization of the three-dimensional (3D)-RI tomograms of EVs (D), the selection of the most representative 2D-RI tomograms of the EVs located in different layers (slices) with isolines representing the regions with the same RI value $\left(\mathbf{D}_{1}\right)$, the quantitative analysis of the significant variations in the reconstructed RI values between EVs $\left(\mathbf{D}_{2}\right)$, separation/splitting of the digitally stained 2D-RI tomograms into red/green/blue channels $(\mathbf{C})$, conversion of the red and blue channels containing different groups of EVs into binary masks $\left(\mathbf{C}_{\mathbf{1}}\right)$, automatic localization and determination of the regions representing EVs $\left(\mathbf{C}_{2}\right)$, determination of the histograms showing the number of EVs with specific diameters $\left(\mathbf{C}_{3}\right)$, and determination of the matrices with the EV size distribution for two groups indicated by RI values $\left(\mathbf{C}_{\mathbf{4}}\right)$. AU, arbitrary unit.

\section{Immunohistochemistry}

Immunohistochemical reactions were performed on formalin-fixed, paraffin-embedded blocks from frozen tissue of nine archival samples included in the study. Whole histologic slides $(4 \mu \mathrm{m}$ thick) from individual CRC patients were used to perform immunohistochemical reactions against Ki-67 (RTU; Dako; IR626; clone MIB-1), CD63
(1:200 dilution; SAB4301607; Sigma-Aldrich), and cytokeratin 20 (RTU; Dako; clone Ks20.8). To detect the coexpression of all biomarkers on each glass slide, a triple staining immunohistochemistry assay was performed (ab183286; Abcam). All reactions, including incubation steps and visualization, were performed following the manufacturer's instructions. Next, the slides were scanned using a Pannoramic MIDI II scanner (3DHistech, Budapest, 


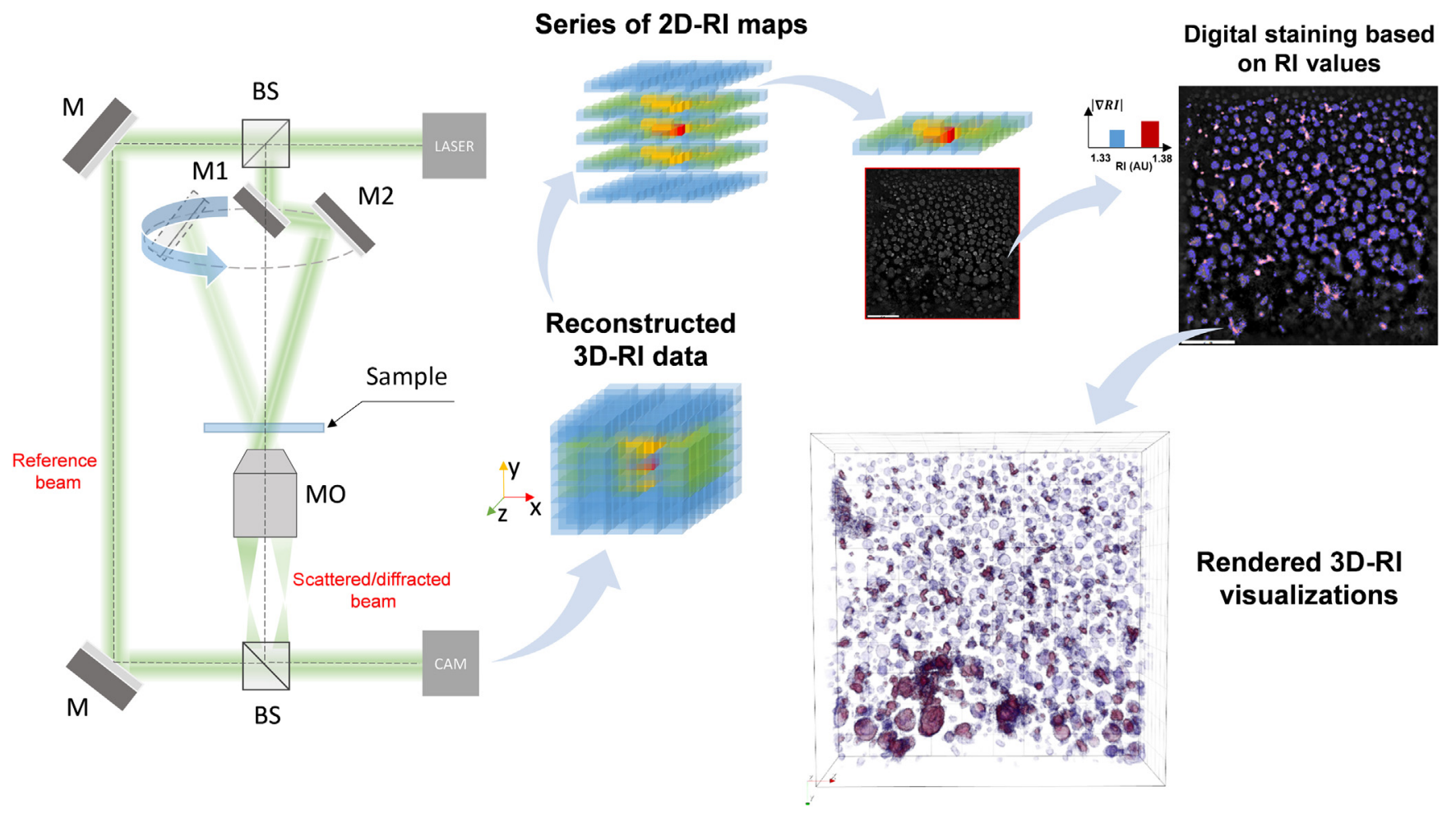

Figure 2 The experimental interferometer system and initial refractive index (RI) data processing. 2D, two dimensional; 3D, three dimensional; $A U$, arbitrary unit; BS, beam splitter; CAM, camera; M, flat mirrors; M1/M2, mirrors on the rotatable arm; M0, microscopic objective.

Hungary). Digital slides were further assessed and processed using CaseViewer software version 2.4 (3DHistech). The exact quantification of Ki-67 expression was performed using QuantCenter (3DHistech) digital image analysis software version 2.1 on chromogenic immunohistochemistry of corresponding tissue microarray cores. To quantify the percentage of positive nuclei among cancer cell clusters, the NuclearQuant (3DHistech) module was used.

\section{DHT Optical System and Measurement Conditions}

DHT is a tomographic version of digital holographic microscopy that enables quantitative phase imaging. In general, digital holographic microscopy enables the measurement of the integrated phase as a product of the optical path-length delay and RI. It is based on the numerical reconstruction of the digital hologram resulting from the interference of the reference beam and the beam diffracted on the object to quantitatively retrieve the $2 \mathrm{D}$ amplitude and phase images of the object (Figure 1). However, in this investigation, to obtain the 3D-RI distribution it was necessary to use the tomographic imaging approach. In recent examinations, a commercial offaxis Mach-Zehnder interferometric setup (Figure 2) with a rotatable scanning mirror (3D Cell Explorer, Nanolive, Switzerland) was used. The examined samples were illuminated by laser beam (wavelength, $520 \mathrm{~nm}$ ) at various incident angles by changing the position of the rotatable arm of the interferometer with the M1 and M2 mirrors. Then, the light beam scattered/diffracted on the sample (object beam) was transmitted through the camera by means of a dry objective microscope $(60 \times$; numerical aperture $=0.8$; Nikon, Tokyo, Japan). The object and reference beam interfered with the camera, and a series of digital holograms were registered for each position of the scanning mirror on the rotatable arm. After the numerical reconstruction ${ }^{23}$ of multiple 2D digital holograms, it was possible to retrieve the RI data in the form of the 3D-RI distribution (3D-RI tomogram) of the examined samples. On the basis of a 2D-RI tomogram at z-position for which the best contrast of EV structures was achieved, digital staining of the EVs was performed, and rendered 3D visualizations of EVs were obtained. For each sample [UC/healthy colon (HC); CRC; cell lines: CCD18Co, HMEC-1, HT-29, CaCo-2, and LoVo; EVs extracted from these cells and EVs extracted from colorectal carcinoma and adenocarcinoma tissues], 15 to 20 3D-RI tomograms were reconstructed. Each 3D-RI tomogram contained 95 slices (2D-RI tomograms) with an axial resolution of $367 \mathrm{~nm}$ and a lateral resolution of $200 \mathrm{~nm}$. A total number of 500 3D-RI tomograms and 47,500 2D-RI tomograms were analyzed. Digital staining of EVs was performed with STEVE software version 1.6.3496 (Ecublens, Switzerland), based on the reconstructed RI values.

Because the EV samples were prepared with different procedures, additional measurements of the RI value of surrounding/mounting reagents were performed with an Abbe refractometer (NAR-2T; minimum scale, 0.001; ATAGO Co. Ltd., Tokyo, Japan) at $20^{\circ} \mathrm{C}$. The RI values for ExoQuick, TEIK, and ultracentrifugation and differential 

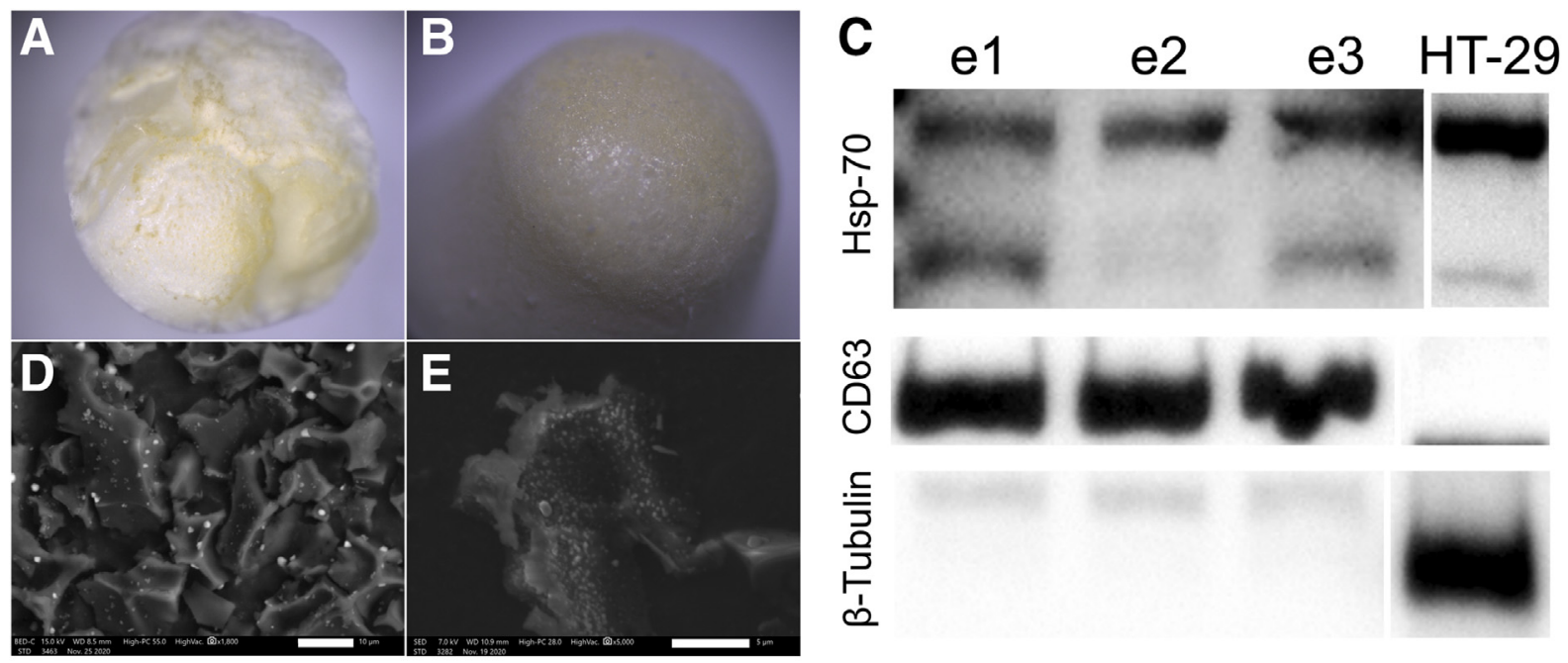

\section{$\mathbf{F}$}

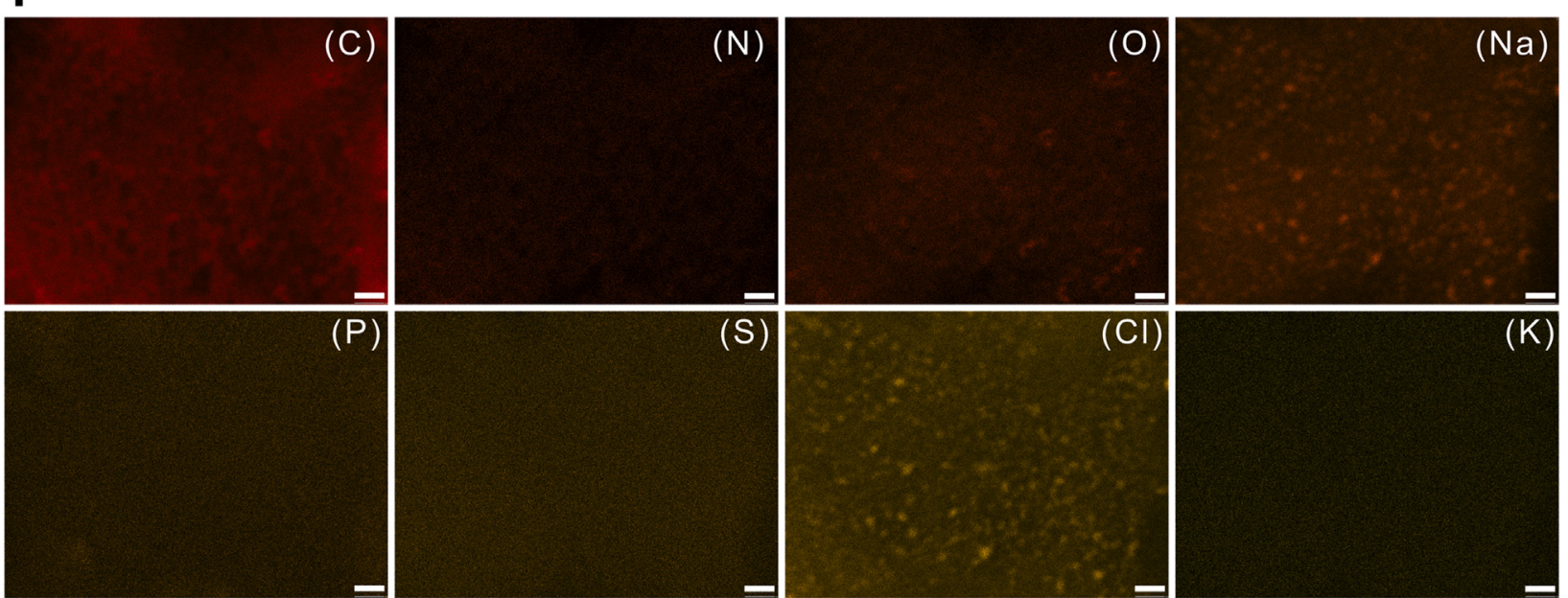

G
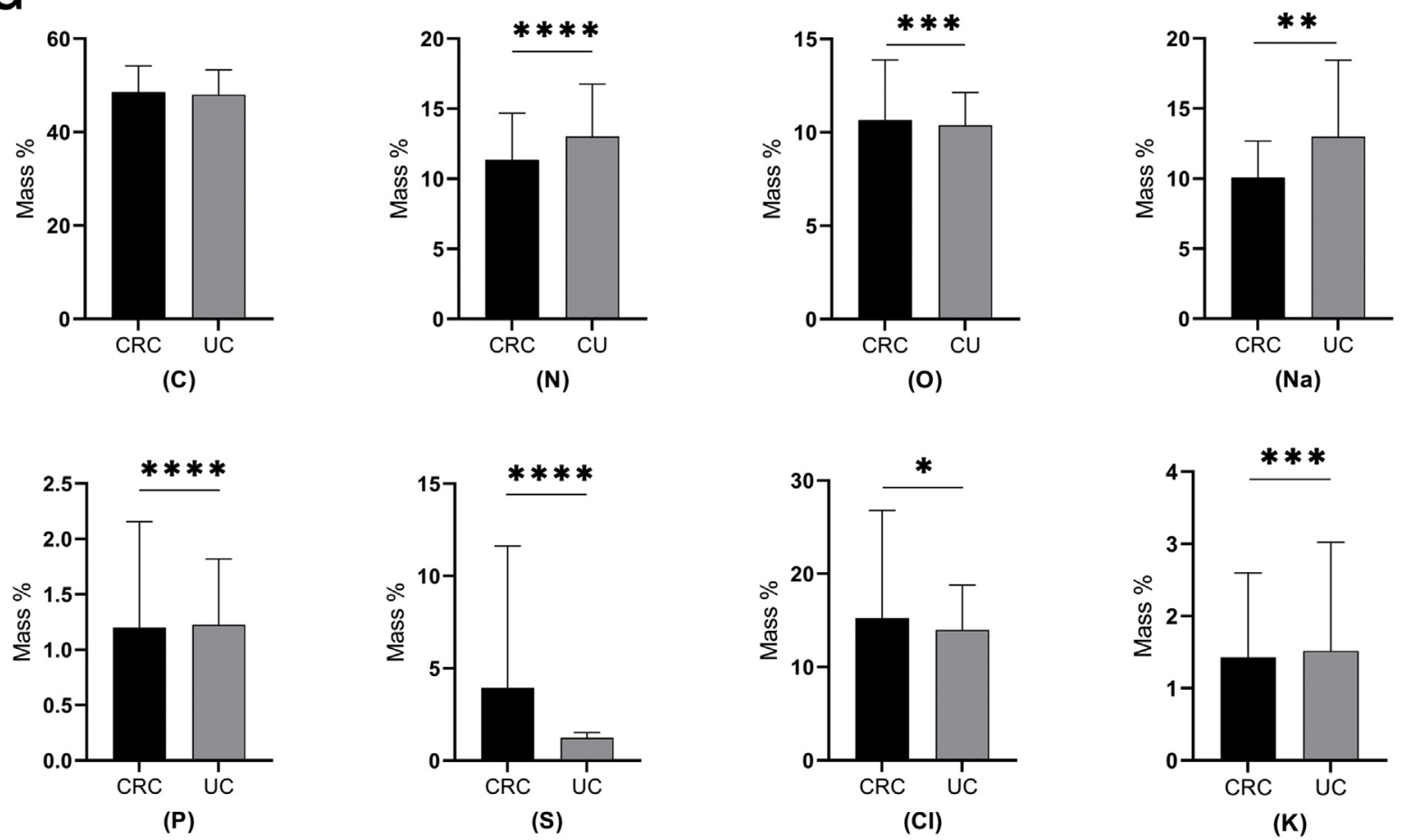
ultracentrifugation liquid phase were $1.3350,1.3770$, and 1.3590 , respectively. The RI values of the media for cell culture were 1.3360 and 1.3365 .

\section{DHT Cell Imaging}

HT-29, CaCo-2, LoVo, CCD-18Co, and HMEC-1 cells were seeded $\left(2 \times 10^{4}\right.$ cells) on glass coverslips (Carl Roth, Carlsruhe, Germany) placed in cell culture Petri dishes (TPP, Trasadingen, Switzerland) and cultured in appropriate medium for 48 hours before DHT imaging. Fresh media were used for the RI control. At the same time, conditioned media samples were collected for EV pellet isolation from the following cell lines at the corresponding numbers of cells: HT-29 $\left(7.2 \times 10^{6}\right)$, CaCo-2 $\left(1.4 \times 10^{6}\right)$, LoVo $\left(4.8 \times 10^{6}\right)$, CCD-18Co $\left(9.0 \times 10^{5}\right)$, and HMEC-1 $\left(1.7 \times 10^{6}\right)$.

\section{Qualitative and Quantitative Examination of EVs Using DHT}

RI data of the reconstructed 2D-RI tomograms and 3D-RI tomograms of EVs were used for qualitative and quantitative analysis to confirm the existence of statistically significant differences between RI values of EVs and determine the vesicle size distribution. A preliminary study concerning the feasibility of differentiating the samples with UC, CRC, and surgically removed healthy margins representing normal colonic mucosa based on EV RI values was performed. However, as previously reported, ${ }^{36}$ the reconstructed RI values were affected by the fixation methods, ${ }^{36}$ which suggests that the sample preparation procedures can significantly affect the RI data. Therefore, DHT examination was performed on samples prepared with different isolation techniques, including the use of commercially available kits (ExoQuick and TEIK reagents), ultracentrifugation (U), and differential ultracentrifugation on a sucrose cushion (DU). Next, to investigate the correlation between the changes with RI values regarding EV size distributions, EV numbers, and clinicopathologic data, DHT examinations were performed on archival frozen samples representing colorectal carcinoma and adenocarcinoma cases with different tumor grades and cell proliferative activities. Then, comparative analysis was performed on cell lines
(CCD-18Co, HMEC-1, HT-29, CaCo-2, and LoVo), and EVs were extracted from those cells.

\section{Analysis of the Differences between the RI Values of Individual EVs}

Considering the highly diverse content of EVs, it can be assumed that individual EVs will exhibit different RI values, which are related to the local variation in their density. After reconstructing the series of 2D-RI tomograms (Figure 1A), they were digitally stained on the basis of RI values of individual EVs present in the examined samples (Figure 1, B and C), enabling the visualization of single EVs (Figure 1D) on the 3D-RI tomograms. Next, the 2D-RI tomograms reconstructed at the best focal plane of each analyzed sample were manually selected from 3D-RI data to determine the slices with the best contrast and to characterize the heterogeneity of the RI-value distribution of single EVs (Figure 1D). The representative 2D-RI distributions of the EVs were used to investigate the possible differentiation of various subtypes of EVs based on their unique RI values. The existence of statistically significant differences between the RI values of EVs was confirmed by one-way analysis of variance (Figure 1D). If the determined $P$ value [probability that the F-statistic can take a value larger than the computed test-statistic value $($ Prob $>F)$ ] for the F-statistic is smaller than the assumed significance level (in this case, 0.05), then the test rejects the null hypothesis that all group means are equal. The normality assumption of the extracted average 3D-RI values of EVs was verified by the Anderson-Darling test. The statistical analysis of the RI was performed using MATLAB R2020a (MathWorks Inc., Natick, MA).

Moreover, the RI values of cells are directly related to the cellular dry mass, which is an important biophysical factor describing the biomass, mass of all cellular content except the water. Therefore, it can offer the more detailed information for EV differentiation based on dry mass of their cargo content. The RI values inside the cell are linearly proportional to its biochemical component, such as proteins, lipids, sugars, and nucleic acids. ${ }^{37}$ For biological applications in cellular objects, the dry mass density $\mathrm{C}(\mathrm{g} / \mathrm{dL})$ can be derived from the RI through a linear calibration model ${ }^{38,39}$ :

$$
R I_{S}(x, y, z)=R I_{H_{2} 0}[\alpha C(x, y, z)+1]
$$

\footnotetext{
Figure 3 A and B: Representative images of exosomal pellets performed under the binoculars from the top layer (A) and from their bottom surface (B). C: Immunoblotting of heat shock protein (Hsp)-70 (53 to $70 \mathrm{kDa}), \mathrm{CD} 63(53 \mathrm{kDa})$, and $\beta$-tubulin $(50 \mathrm{kDa})$ levels in exosomal pellets (e1, e2, and e3) and archival HT-29 cell line. The analyses were performed on pellets obtained from human plasma microsamples (e1 and e3, colorectal cancer; and e2, ulcerative colitis). An Invitrogen kit was used to precipitate EVs. D and E: Scanning electron microscopy (SEM) micrographs visualized the extracellular vesicles (EVs) in the examined pellets. EVs with different morphologic parameters were present on both the surface of the pellet (D) and inside the crystallized masses of the sample (E). F: Representative SEM-energy dispersive spectrometer detector (EDS) mapping of the superficial layer of the lyophilized exosomal pellet (ulcerative colitis) that displays distinct compound of chemical elements for individual extracellular vesicles: carbon (C), nitrogen (N), oxygen ( 0$)$, sodium (Na), phosphorus $(\mathrm{P})$, sulfur $(\mathrm{S})$, chlorine $(\mathrm{Cl})$, and potassium $(\mathrm{K})$. The organic content of visualized EVs was verified. Interestingly, larger particles appear to be richer in salt content, showing higher distribution of sodium and chlorine. For semiquantitative EDS analysis, the single EVs detected in the selected scanning area of exosomal pellets were marked as single spots, counted, and then measured of their individual spectral peaks. G: SEM-EDS analysis was performed for each of the above-mentioned chemical elements on EVs isolated from colorectal cancer (CRC) and ulcerative colitis (UC) samples. The normalized weight percentages for each element were taken into consideration (Mass \%). The elemental composition of exosomal pellets differs in regard to diagnosis. $n=2$ CRC and UC samples (G). ${ }^{*} P \leq 0.05,{ }^{* *} P<0.01,{ }^{* *} P<0.001$, and ${ }^{* * *} P<0.0001$. Scale bars: $10 \mu \mathrm{m}(\mathbf{D}) ; 5 \mu \mathrm{m}(\mathbf{E}) ; 1 \mu \mathrm{m}(\mathbf{F})$.
} 
A
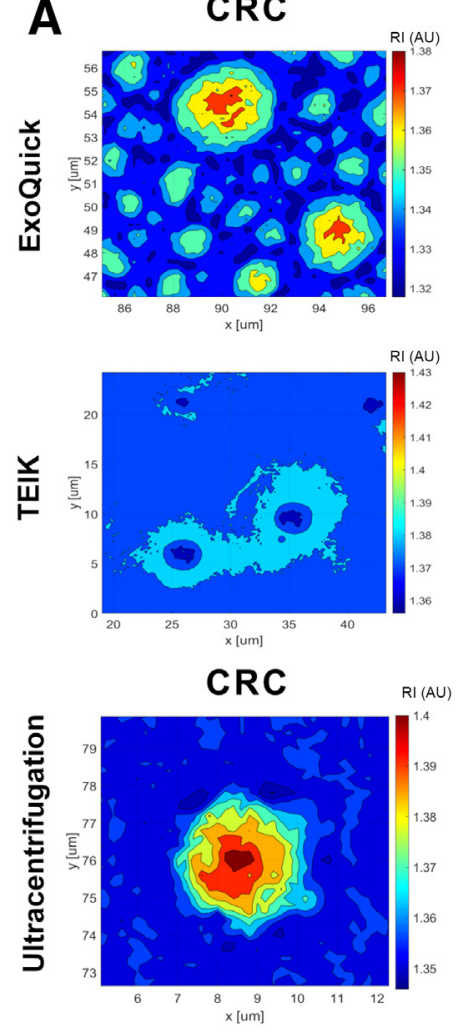

UC
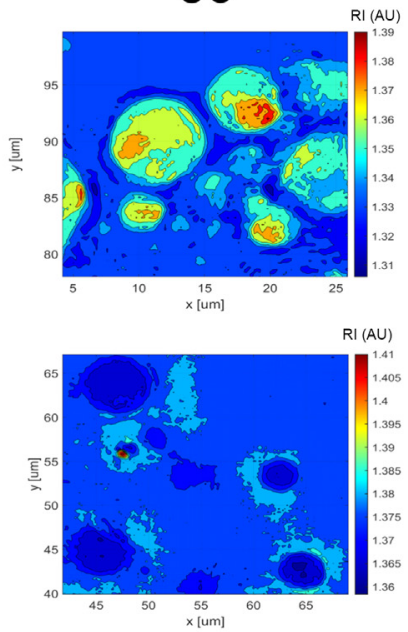

HC

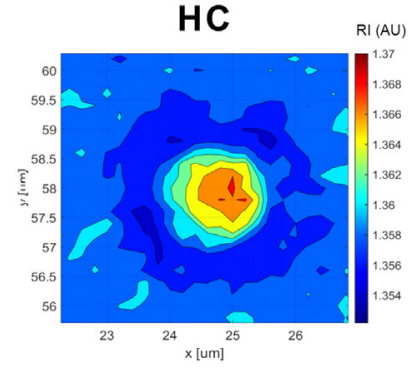

B
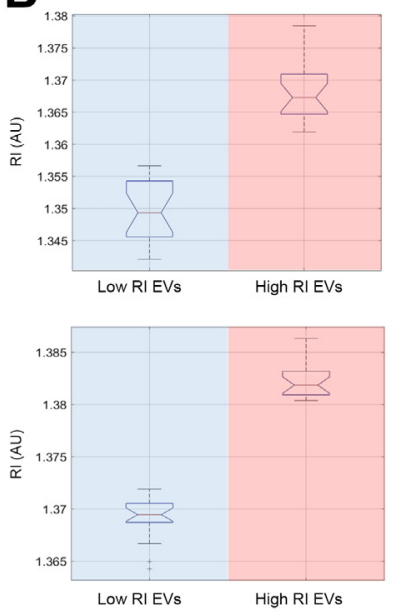

CRC

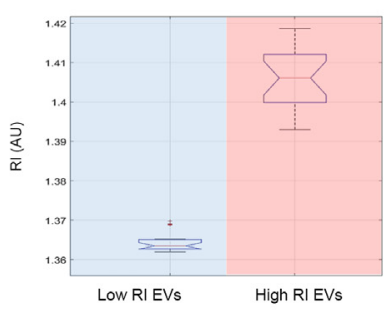

UC
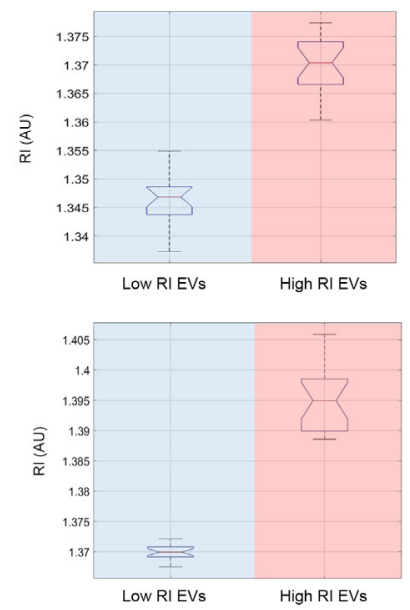

HC

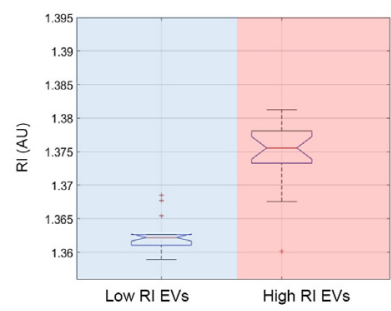

Figure 4 Representative two-dimensional-refractive index (RI) tomograms of extracellular vesicles (EVs), demonstrating the spatial heterogeneity of RI distributions. A: Colorectal cancer (CRC), ulcerative colitis (UC), and healthy surgical margin [healthy colon (HC)] samples were prepared with different isolation procedures. Comparison of RI value differences between EVs precipitated from different tissues and methods of isolation. B: The representative boxplots demonstrating the variation of the RI values among two determined RI ranges (low RI and high RI), enabling the differentiation of individual EVs isolated from CRC, UC, and HC. Red shading corresponds to the EVs with higher RI values, whereas blue shading corresponds to the EVs with lower RI values. $\mathrm{AU}$, arbitrary unit; TEIK, Total Exosome Isolation Kit.

where $\alpha$ is the proportionality constant called the RI increment. For biological cells, this coefficient values are changing, with ranges from 0.0018 to $0.0021 \mathrm{dL} / \mathrm{g} .{ }^{40}$ Cells such as erythrocytes are assigned the specific value $\alpha=0.0019 \mathrm{dL} / \mathrm{g}$, because they highly enrich hemoglobin.
However, as referred previously, ${ }^{37}$ the constant $\alpha$ equal to $0.0018 \mathrm{dL} / \mathrm{g}$ covers not only proteins, but also lipids, sugars, and nucleic acid content. Hence, in this study, this value was used to assess the complexity of EV cargo. The dry mass was determined from the above equation based on data

Table 2 The Determined Mean $\mathrm{RI}_{\mathrm{B}}$ and the Mean $\mathrm{RI}_{\mathrm{HIGH}}$ and $\mathrm{RI}_{\mathrm{LOW}}$ of EVs

\begin{tabular}{|c|c|c|c|c|}
\hline Method & $\mathrm{RI}_{\mathrm{HIGH}}$, means $\pm \mathrm{SD}, \mathrm{AU}$ & $\mathrm{RI}_{\mathrm{Low}}$, means $\pm \mathrm{SD}, \mathrm{AU}$ & $\Delta \mathrm{RI}_{\mathrm{HIGH}-\mathrm{LOW}}, \mathrm{AU}$ & $\mathrm{RI}_{\mathrm{B}}$, means $\pm \mathrm{SD}$ \\
\hline ExoQuick & & & & $1.33502 \pm 0.00026$ \\
\hline UC & $1.37031 \pm 0.00491$ & $1.34633 \pm 0.00421$ & 0.02398 & \\
\hline CRC & $1.36821 \pm 0.00472$ & $1.35001 \pm 0.00483$ & 0.01820 & \\
\hline TEIK & & & & $1.37710 \pm 0.00569$ \\
\hline UC & $1.39524 \pm 0.00501$ & $1.36991 \pm 0.00134$ & 0.02533 & \\
\hline CRC & $1.38242 \pm 0.00182$ & $1.36910 \pm 0.00204$ & 0.01332 & \\
\hline CRC & $1.40643 \pm 0.00321$ & $1.36361 \pm 0.00257$ & 0.04282 & \\
\hline Differential ultracentrifugation & & & & $1.35896 \pm 0.00191$ \\
\hline $\mathrm{HC}$ & $1.36763 \pm 0.00443$ & $x$ & $x$ & \\
\hline CRC & $1.41542 \pm 0.00343$ & $x$ & $x$ & \\
\hline
\end{tabular}

$\mathrm{AU}$, arbitrary unit; $\mathrm{CRC}$, colorectal cancer; $\mathrm{EV}$, extracellular vesicle; $\mathrm{HC}$, healthy colon; $\mathrm{RI}_{\mathrm{B}}$, refractive index of the surrounding medium/background; $\mathrm{RI}_{\mathrm{HIGH}}$ higher refractive index; $\mathrm{RI}_{\mathrm{Low}}$, lower refractive index; TEIK, Total Exosome Isolation Kit; UC, ulcerative colitis; $\mathrm{X}$, lack of results due to absence of low-RI fraction of EVs. 
Table 3 The Results of One-Way ANOVA

\begin{tabular}{|c|c|c|c|c|c|c|}
\hline Method & Source of variability & SS & $d f$ & MS & $\mathrm{F}$ & Prob $>F$ \\
\hline \multirow[t]{5}{*}{ ExoQuick } & CRC & & & & & \\
\hline & Group (between) & 0.00333 & 1 & 0.00333 & 1909.96 & $1.4333 \times 10^{-14}$ \\
\hline & Total & 0.0042 & 500 & & & \\
\hline & UC & & & & & \\
\hline & Group (between) & 0.00577 & 1 & 0.00577 & 3644.59 & $4.6338 \times 10^{-19}$ \\
\hline \multirow[t]{5}{*}{ TEIK } & CRC & & & & & \\
\hline & Group (between) & 0.00175 & 1 & 0.00175 & 6237.5 & $3.1441 \times 10^{-23}$ \\
\hline & Error (within) & 0.00014 & 498 & 0.00001 & & \\
\hline & Total & 0.00189 & 500 & & & \\
\hline & UC & & & & & \\
\hline \multirow{7}{*}{ Ultracentrifugation } & Group (between) & 0.00692 & 1 & 0.00692 & 5660.79 & $3.2314 \times 10^{-22}$ \\
\hline & Error (within) & 0.00061 & 498 & 0.00001 & & \\
\hline & Total & 0.00753 & 500 & & & \\
\hline & $\mathrm{HC}$ & & & & & \\
\hline & Group (between) & 0.00258 & 1 & 0.00258 & 4152.97 & $4.2131 \times 10^{-22}$ \\
\hline & Error (within) & 0.00031 & 498 & 0.00001 & & \\
\hline & Total & 0.00289 & 500 & & & \\
\hline
\end{tabular}

ANOVA, analysis of variance; CRC, colorectal cancer; df, degree of freedom associated with each source; F, F statistics; HC, healthy colon; MS, mean squares for each source; Prob $>F, P$ value that is the probability that F-statistic can take a value larger than computed F-statistic value; $S S$, sum of squares due to each source; TEIK, Total Exosome Isolation Kit; UC, ulcerative colitis.

about the dry mass density-concentration of their solids derived from reconstructed RI values and their volumes, which were obtained from the reconstructed 3D-RI distributions.

\section{Analysis of the Size Distribution of Single EVs with RI Values}

To characterize the size distribution of the EVs with different RI values (Figure 1), a series of 2D-RI tomograms were digitally stained on the basis of the determined RI values of the two EV groups and the surrounding reagent. Then, the stained 2D-RI tomograms were separated on three channels: red corresponded to the EVs with higher RI values, blue corresponded to EVs with lower RI values, and green corresponded to the liquid phase surrounding the examined vesicles (Figure 1C). Next, to assess EV size distribution, only the red and green channels after contrast enhancement were used, and they were converted to binary masks (Figure 1C). After that, the initial evaluation of the range of EV diameters and the determination of the circularity parameter of individual EV regions were extracted, and their size was determined (Figure 1C). The circularity parameter ranges from 0 to 1 , where 1 indicates a perfect circle, and 0 indicates an elongated shape. In this approach, the circularity parameter ranged from
0.8 to 1.0. Next, the diameters of EVs were determined, and histograms representing the distribution of the number of EVs with specific diameters were generated. For each histogram, the probability density function, based on the inverse gaussian distribution (or Wald distribution) with support on $(0, \infty)$, was fitted (Figure 1C). Then, matrices representing the distribution of EV diameters with lower and higher RI values for all analyzed samples were determined (Figure 1C). All 2D-RI tomogram processing was performed in MATLAB $\mathrm{R} 2020 \mathrm{a}$, except for the extraction of the EV regions, automatic counting, and determination of the EV size, which were performed with ImageJ software version $1.53 \mathrm{i}^{41}(\mathrm{NIH}$, Bethesda, MD; https://imagej.nih.gov/ij).

\section{Scanning Electron Microscopy}

The imaging and chemical composition analyses were performed using a JEOL JSM-IT500LA InTouchScope scanning electron microscope (JEOL Ltd, Tokyo, Japan) with tungsten fiber as the electron source. SEM was used to visualize EVs in the examined pellet samples. SEM micrographs were taken in the high-resolution mode with a backscattered electron detector. The acceleration voltage and working distance were individually adjusted for every image. Each sample was dried by lyophilization before 

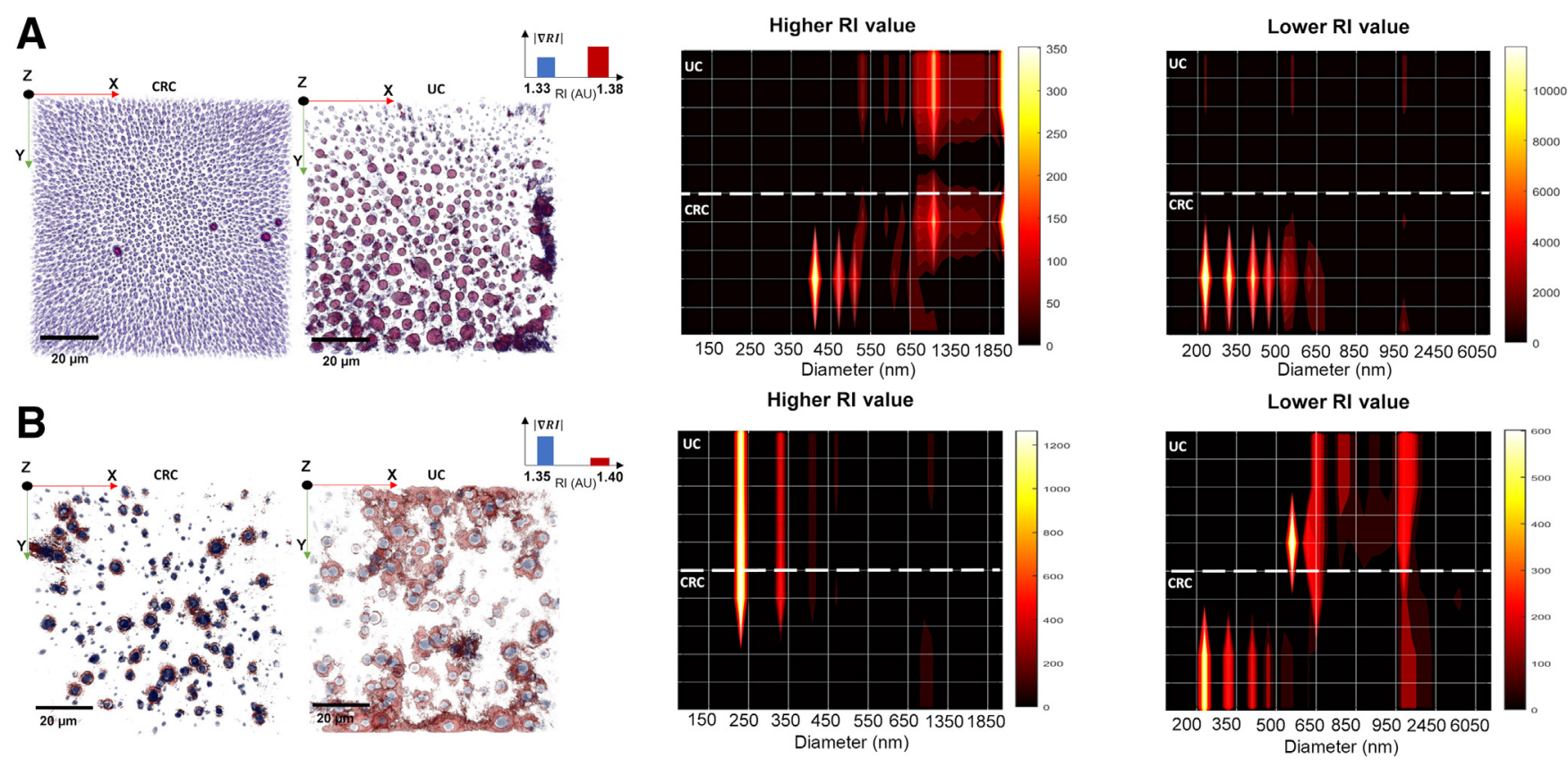

C
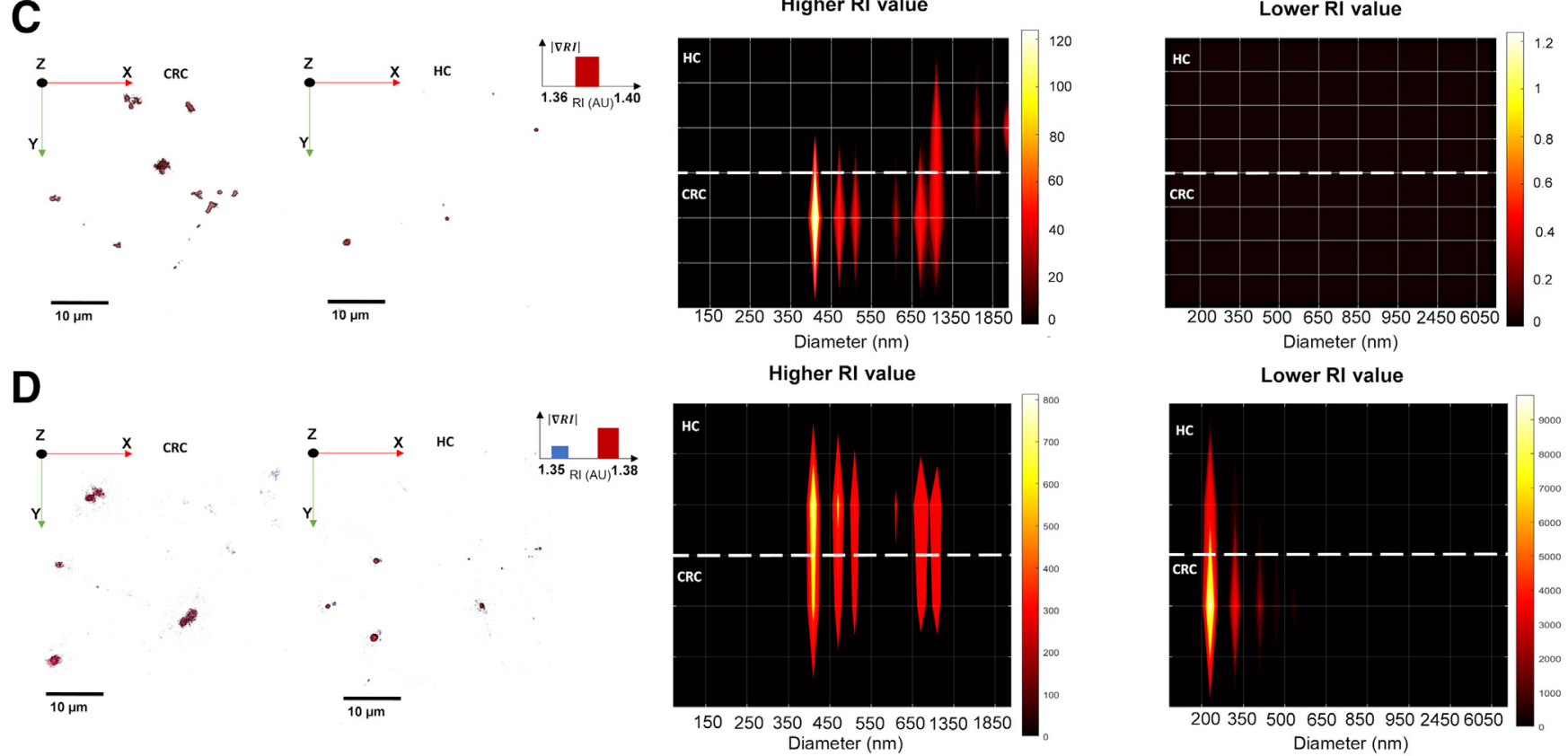

Figure 5 The influence of the preparation procedures [ExoQuick, Total Exosome Isolation Kit (TEIK), ultracentrifugation (U), and differential ultracentrifugation on a sucrose cushion (DU)] on refractive index (RI) data and size distributions of extracellular vesicles (EVs). The visualization results containing the rendered three-dimensional-RI tomograms (left sides) of EVs and the matrices (right sides) showing the distribution of EV diameters in the two RI fractions (lower/higher RI) obtained for samples prepared with the ExoQuick (A), TEIK (B), U (C), and DU (D) procedures. Color bar showing the number of EVs. Dashed white lines separate the EVs' diameter's distributions of the two different analyzed samples: ulcerative colitis (UC)/colorectal cancer (CRC) and healthy colonic mucosa (HC)/CRC. Scale bars: $20 \mu \mathrm{m}$ (A and B); $10 \mu \mathrm{m}$ (C and D). AU, arbitrary unit.

SEM imaging. Samples for SEM were freeze dried in a vacuum freeze dryer at $-54^{\circ} \mathrm{C}$. The mass that was left after freeze drying was glued with carbon tape and imaged in this form by SEM. Analysis of the chemical composition of EVs was performed with an energy dispersive spectrometer detector (EDS) using SMILE VIEW LAB Application Data Management software version 3.1.3.9 (Jeol, Tokyo, Japan).

\section{Results}

\section{Verification of the Exosomal Nature of Examined} Samples

EVs were identified in SEM micrographs of the prepared pellets visible under the binocular (Figure 3, A, B, D and E). Western blot analysis showed the presence of heat shock 
Table 4 The Percentage (Relative SD) Distribution of the Diameters of EVs Present in the Samples Prepared According to ExoQuick and TEIK Procedures

\begin{tabular}{|c|c|c|c|c|c|c|c|c|}
\hline \multirow{3}{*}{$\begin{array}{l}\text { EV diameter } \\
\text { range, } \mathrm{nm}\end{array}$} & \multicolumn{4}{|c|}{ ExoQuick, \% (relative SD) } & \multicolumn{4}{|c|}{ TEIK, \% (relative SD) } \\
\hline & \multicolumn{2}{|l|}{ CRC } & \multicolumn{2}{|l|}{ UC } & \multicolumn{2}{|l|}{ CRC } & \multicolumn{2}{|l|}{ UC } \\
\hline & $\mathrm{RI}_{\text {LoW }}$ & $\mathrm{RI}_{\mathrm{HIGH}}$ & $\mathrm{RI}_{\text {LoW }}$ & $\mathrm{RI}_{\mathrm{HIGH}}$ & $\mathrm{RI}_{\text {LoW }}$ & $\mathrm{RI}_{\mathrm{HIGH}}$ & $\mathrm{RI}_{\text {LoW }}$ & $\mathrm{RI}_{\mathrm{HIGH}}$ \\
\hline $500-1000$ & $24.21(1.23)$ & $20.45(1.67)$ & $41.14(1.52)$ & $63.18(1.56)$ & $24.74(1.78)$ & $20.37(1.88)$ & $76.06(1.23)$ & $12.26(0.97)$ \\
\hline$>1000$ & $2.53(0.23)$ & $4.68(1.87)$ & $32.32(1.65)$ & $27.76(1.51)$ & $26.72(1.84)$ & $8.58(1.76)$ & $23.94(1.35)$ & $1.30(1.12)$ \\
\hline
\end{tabular}

$\mathrm{CRC}$, colorectal cancer; $\mathrm{EV}$, extracellular vesicle; $\mathrm{RI}_{\mathrm{HIGH}}$, higher refractive index; $\mathrm{RI}_{\mathrm{LOw}}$, lower refractive index; TEIK, Total Exosome Isolation Kit; UC, ulcerative colitis.

protein 70 and CD63 antigens, which are considered specific markers for these nanoparticles. The negative control was the HT-29 cell line (archival cell pellet), which was positive for $\beta$-tubulin and negative for $\mathrm{CD} 63$ protein expression (Figure $3 \mathrm{C}$ ). The samples were verified according to the International Society for Extracellular Vesicles guidelines. $^{42}$

\section{EDS Analysis of Single EVs Reveal the Heterogeneity in the Composition of Chemical Elements between CRC and UC}

The exosomal pellets $(n=4)$ isolated from human plasma from patients diagnosed with CRC $(n=2)$ and UC $(n=2)$ were used for further SEM-EDS analysis. EVs were precipitated using the ExoQuick method by adding $63 \mu \mathrm{L}$ of reagent to $250 \mu \mathrm{L}$ of total plasma per sample. Samples were incubated for 1 hour at $4^{\circ} \mathrm{C}$. Next, the samples were carefully transported on ice and cryodesiccated in accordance with a previously described protocol (Scanning Electron Microscopy). The aim of EDS analysis was to detect subtle differences in the distribution of selected biogenic chemical elements at the single-vesicle level. Regions of interest were selected by selecting the most representative areas of pellets targeted at an initial magnification of $\times 2000$ to $\times 5000$. Single EVs were detected, estimated, and counted at a sufficiently higher magnification most suitable for each examined area $(\times 10,000)$. EDS spectra were collected from the most prominent single EVs to quantify the distribution of the following chemical elements: carbon, nitrogen, oxygen, sodium, phosphorus, sulfur, chlorine, and potassium. The working distance was set to $10.1 \mathrm{~mm}$, and the semiquantitative ZAF method was used. The results showing the exact chemical element distributions for individual EVs were normalized to $100 \%$. Finally, spectra were obtained from 241 cancer-associated EVs and 184 EVs from UC patients (Figure 3G). Statistical analysis was performed using GraphPad Prism software version 8.1.2 (GraphPad Software Inc., San Diego, CA) and $U$-tests (for two group comparisons). $P<0.05$ (two tailed) was considered statistically significant. The greatest differences in the distribution level were noted for sulfur, nitrogen, and phosphorus, wherein the percentage content of those elements significantly differed between UC and CRC $(P<0.0001)$. For colitis patients, the mean percentage distribution of sulfur was $1.239 \%$ (SD, $0.2810 \%$ ); and for CRC patients, its chemical composition was significantly higher (mean, 3.940\%; SD, 7.690\%). In contrast, the mean of phosphorus for CRC was significantly decreased $(1.199 \%$; SD, $0.9560 \%)$ compared with that for UC (1.225\%; SD, 0.5948\%). A similar trend was observed for nitrogen, where its composition was higher in colitis (mean, 13.03\%; SD, 3.719\%) than in cancer (mean, 11.32\%; SD, 3.354\%). The distribution of sodium was significantly higher in UC patients (mean, 12.99\%; $\mathrm{SD}, 5.468 \%$ ) than in CRC patients (mean, 10.2\%; $\mathrm{SD}, 2.657 \% ; P=0.0025)$. Interestingly, the distribution

Table 5 The Percentage Distribution (Relative SD) of the Diameter of EVs Present in the Samples Prepared According to U and DU Procedures

\begin{tabular}{|c|c|c|c|c|c|c|c|c|}
\hline \multirow{3}{*}{$\begin{array}{l}\text { EV diameter } \\
\text { range, } \mathrm{nm}\end{array}$} & \multicolumn{4}{|c|}{ U, \% (relative SD) } & \multicolumn{4}{|c|}{ DU, \% (relative SD) } \\
\hline & \multicolumn{2}{|l|}{ CRC } & \multicolumn{2}{|l|}{$\mathrm{HC}$} & \multicolumn{2}{|l|}{ CRC } & \multicolumn{2}{|l|}{$\mathrm{HC}$} \\
\hline & $\mathrm{RI}_{\text {LoW }}$ & $\mathrm{RI}_{\mathrm{HIGH}}$ & $\mathrm{RI}_{\text {LoW }}$ & $\mathrm{RI}_{\mathrm{HIGH}}$ & $\mathrm{RI}_{\text {LoW }}$ & $\mathrm{RI}_{\mathrm{HIGH}}$ & $\mathrm{RI}_{\text {Low }}$ & $\mathrm{RI}_{\mathrm{HIGH}}$ \\
\hline $500-1000$ & $4.31(1.23)$ & $47.95(1.21)$ & $4.58(1.41)$ & $47.24(1.10)$ & $0.00(0.00)$ & $31.04(1.12)$ & $0.00(0.00)$ & $0.00(0.81)$ \\
\hline$>1000$ & $0.00(0.00)$ & $13.79(1.21)$ & $0.00(0.00)$ & $11.46(1.11)$ & $0.00(0.00)$ & $14.94(1.31)$ & $0.00(0.00)$ & 100.00 (1.95) \\
\hline
\end{tabular}

CRC, colorectal cancer; DU, differential ultracentrifugation; EV, extracellular vesicle; $H C$, healthy colon; $\mathrm{RI}_{\mathrm{HIGH}}$, higher refractive index; $\mathrm{RI}_{\mathrm{Low}}$, lower refractive index; U, ultracentrifugation. 


\section{A}

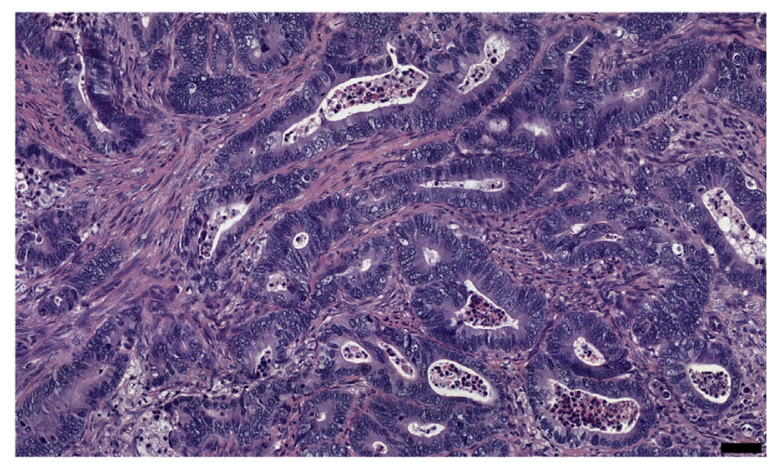

G3

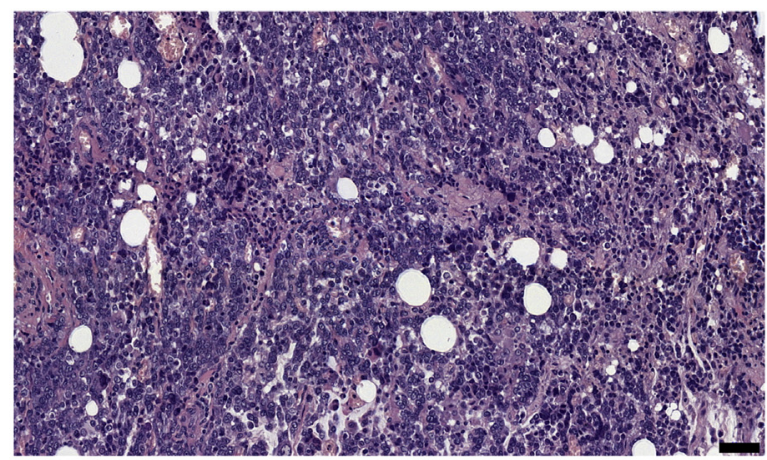

C

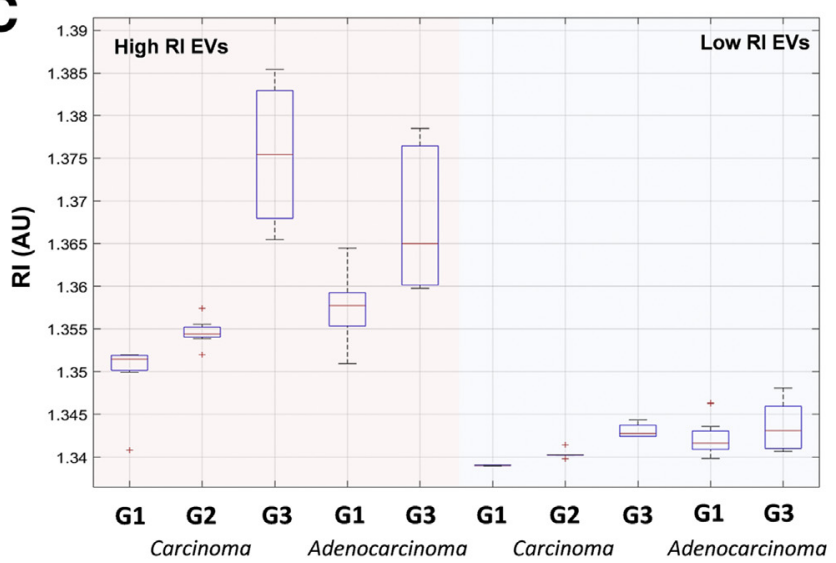

E
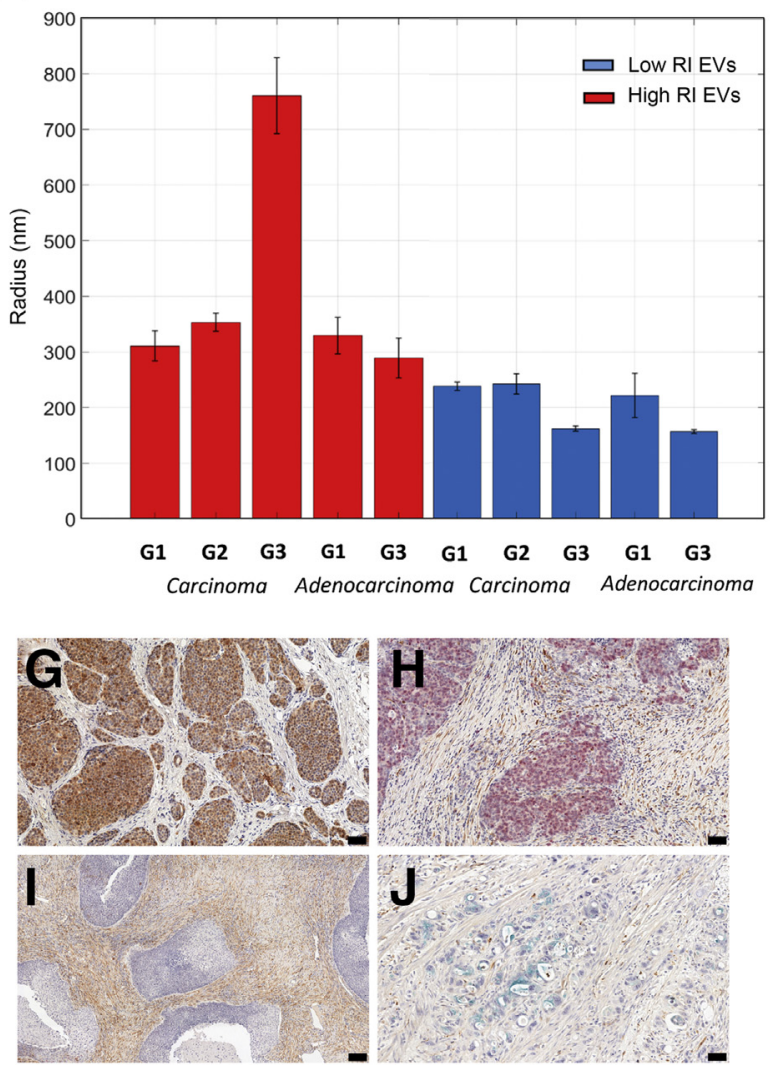
of chlorine was slightly higher in CRC patients (mean, $15.23 \%$; SD, $11.57 \%$ ) than UC patients (mean, $13.96 \%$; $\mathrm{SD}, 4.824 \%$ ), and the difference was significant $(P=0.0424)$. The potassium concentration was higher in CRC (mean, 1.623\%; SD, 1.114\%) than in UC (mean, $1.519 \% ; \mathrm{SD}, 1.504 \% ; \quad P=0.0006)$. The percent composition of oxygen in exosomal pellets was higher in CRC (mean, 10.63\%; SD, 3.259\%) and significantly decreased in UC (mean, 10.38\%; SD, 1.758\%; $P=0.0006$ ). No significant differences were found for carbon distribution between the tested groups of patients $(P=0.0502)$. Representative EDS maps and a graphical representation of the results are shown in Figure 3F.

\section{D-RI Distributions of Single EVs Exhibit Spatial Heterogeneity Depending on the Diagnosis, Isolation Procedure, and Reagents Used}

Representative results shown in Figure 4 demonstrate the spatial variations in RI values among EVs isolated from plasma microsamples (CRC/UC) and solid tissue isolates (CRC/HC) prepared differently (U/DU). The local variations in RI values of EVs indicate the high heterogeneity of their optical properties. They also confirmed that changes in EV density were related to individual RI values, which can indicate unique fluctuations in the content of EV cargo depending on the type of samples examined (CRC and UC or CRC and HC) and EV size. Reconstructed RI values of EVs can be significantly affected by the sample preparation procedure. In the case of the ExoQuick precipitation kit, $\mathrm{U}$ and DU procedures, the range of the EV RI value variations was higher than that of the RI values of the liquid phase above the isolated pellet; however, for TEIK, a fraction of EVs had lower RI values. This phenomenon was more commonly observed with larger EVs than with smaller EVs (TEIK/UC) (Figure 4), and indicated in the following section. Moreover, the EVs were often trapped (TEIK/CRC) (Figure 4) in the fractions of the reagent used, which acted as a mounting phase and had significantly higher RI values. These observations were also confirmed by SEM examination (grayscale images of EVs trapped under the reagent layer) (Figure 3). Furthermore, the local changes in the RI of the reagent used may have led to errors in the RI reconstruction algorithm due to the mismatch between the local and average/reference RI of the surrounding environment, because the RI values of the examined particles were determined by the relative changes in the RI of the reagent surrounding the individual vesicles. This effect may limit the repeatability and comparability of the obtained results. Table 2 indicates that the SD of the mean RI value was the highest for TEIK procedure. Moreover, in contrast to EVs from samples prepared with other procedures (the DU method), only one fraction of EVs with higher RI values was present.

\section{RI as an Optical Parameter for Categorizing EVs into Two Independent Subtypes}

The examination of the RI value variation inside the single EVs in 2D-RI tomograms (EV slices) showed that for each sample, it was possible to determine two ranges of RI values estimated as low and high to enable the digital staining of all regions (voxels of the 3D-RI tomogram) of EVs present in the samples (Table 2). These RI ranges are used to investigate the differentiation of single EVs and analyze their size distribution, assuming that vesicles with the same RI values have the same content in their cargo. To indicate if the RI ranges were set appropriately, one-way analysis of variance was applied to confirm that the significant changes between the determined RI-value ranges enabled the differentiation of EVs. Representative boxplots showing the differences between the variation and median of the two groups of EVs, characterized by the two ranges of RI values, are shown in Figure 4. Changes in the RI values defining the two RI ranges enabled the differentiation of EVs and did not overlap. Moreover, in each case, the two medians were significantly different at the 5\% significance level, as the notches did not overlap. This difference between the medians and means of each defined RI range indicates a significant difference between the density of individual EVs with lower and higher RI values, which can be correlated with the differences in their unique content. Furthermore, there were also differences between the determined RI value ranges of EVs from UC and CRC samples. However, the observed differences were

\footnotetext{
Figure 6 A: Routine hematoxylin and eosin staining micrographs, showing human primary colorectal adenocarcinomas with different tumor grade (G): $G 1$ stage indicates well-differentiated clusters of cancer cells, and G3 indicates poor-differentiated lesions. B-E: Refractive index (RI) data-based characterization of the heterogeneity of extracellular vesicles (EVs) extracted from solid tissue colorectal cancer samples: exemplary three-dimensional-RI distribution of EVs extracted from adenocarcinomas (G3; B), variation in the RI values for the high and low RI fractions of EVs (C), average dry mass density (D), and average radius of EVs from each (low/high RI) subtype of EVs (E). RI values for individual EVs increased with the tumor grade (C). The extracellular vesicles of higher RI value had increased dry mass density compared with those of lower RI. Dry mass density increased for cancers with higher tumor grade (D). F-J: Triple staining immunohistochemistry against cytokeratin-20 (CK20), Ki-67 (clone MIB-1), and CD63 tetraspanin. Different chromogens were used for visualization of individual biomarkers: CK20 (emerald chromogen; green color), Ki-67 (permanent red chromogen; red color), and CD63 (3,3'-diaminobenzidine; brown color). The immunohistochemical reactions were performed on whole glass slides with two main histopathologic subtypes of colorectal cancer defined as carcinomas and adenocarcinomas for all cases of which frozen tissue material was previously used to perform EV isolates. CD63 antigen as an exosomal marker was predominantly expressed in most of the examined cases; however, the expression pattern differed regarding the tissue area and its co-expression with other biomarkers. $\mathbf{G}$ and I: Representative staining against CD63, showing dominant tumoral (G) and stromal expression (I). $\mathbf{F}$ and $\mathbf{H}$ : Common observed phenomenon was the co-expression between CD63 tetraspanin and Ki-67 antigen in cancer cells. J: Almost all examined cases were CK20 negative, and the positive reactions against that biomarker were detected mostly in the minor areas of adjusted normal colonic mucosa or CK20 had only focal expression in cancer cells for those tumors that were positive. Scale bars $=50 \mu \mathrm{m}(\mathbf{A}$ and $\mathbf{F}-\mathbf{J})$. AU, arbitrary unit.
} 
dependent on the sample preparation procedures. For the ExoQuick and TEIK reagents, $\Delta \mathrm{RI}$ high-low was higher for EVs from UC samples, whereas for the U procedure, the opposite was observed, as indicated for the different types of tissue used to precipitate the exosomal pellets. This was also confirmed by the analysis of variance results (Table 3). For each sample and each preparation procedure, two groups of data were identified: one with RI values corresponding to a low RI fraction of EVs and a second with RI values corresponding to a high RI fraction of EVs. The estimated $P$ values for the F-statistic were significantly lower than the significance level (0.05), which indicates that the test rejected the null hypothesis that all group means were equal. Moreover, the RI variability due to the differences among the group means (variability between groups) was more significant than variability due to the differences between the data in each group and the group mean. This indicates that there was a significant difference between the analyzed EV groups with lower and higher RI values, whereas the RI variability within groups indicated the repeatability of the determined RI values of examined EVs. For each isolation procedure, two different fractions of EVs with significant differences between their RI values were identified. Therefore, regardless of the isolation procedure, it was possible to determine the ranges of individual RI values, which can be used for the digital staining of single EVs.

\section{Assessment of the Heterogeneity of the Size Distribution of EVs with Different RI Values in Plasma Samples}

The identification of RI ranges (low/high) to characterize individual EVs in examined samples enabled their use in digital staining and the analysis of the size distribution at the single vesicle level of the subpopulations with low or high RI values. After the digital extraction of the EVs from the two fractions and determination of their size according to the previously described method (Figure 1), it was possible to generate a histogram showing the distribution of the EV diameters (Supplemental Figures S1 and S2). The mean diameters of EVs in each cohort were determined on the basis of the fitted probability density function. The resulting histogram was used to more intuitively represent the distribution of EVs in the form of matrices, which were compiled with the representative rendered 3D-RI tomograms of examined samples prepared with different procedures (Figure 5). Considering the heterogeneous distribution of EV size, in the matrices, nonlinear diameter value variation was used. For the CRC samples (ExoQuick), EVs with higher RI values had diameters $>410 \mathrm{~nm}$, but most of them had sizes in the following ranges: 410 to 520,650 to 1350 , and $>1850 \mathrm{~nm}$ (Figure $5 \mathrm{~A})$. EVs from UC samples had diameters $>575 \mathrm{~nm}$, while most vesicles had diameters in the following ranges: 525 to 540,565 to 590,625 to 640,650 to 1350 , and $>1850 \mathrm{~nm}$.
EVs with higher RI values from CRC samples showed a much more heterogeneous distribution of diameters than EVs from UC samples, which contained EVs with a larger diameter. For EVs with lower RI values, the diameters of EVs from CRC samples ranged from 205 to $650 \mathrm{~nm}$, whereas for UC samples, the number of EVs was decreased, but some EV fractions with larger diameters (in the ranges of 210 to 220,515 to 525 , and 960 to $980 \mathrm{~nm}$ ) could be distinguished. For both EVs with lower and higher RI values, the smallest EVs were identified in CRC samples. The same trend was observed for EVs with lower RI values from both UC and CRC samples (TEIK), where most EVs isolated from colorectal adenocarcinoma samples had diameters in the range of 200 to $500 \mathrm{~nm}$, whereas most of UC EVs had larger diameters $(>510 \mathrm{~nm})$ (Figure 5B). Most EVs with higher RI values had diameters from 230 to $410 \mathrm{~nm}$ for both UC and CRC samples. However, the smaller EVs with higher RI values formed clusters, which may have been caused by their entrapment in the mounting reagent, as indicated in the previous section.

For HC and CRC samples prepared with the $\mathrm{U}$ procedure (Figure 5, C and D), there was no significant difference between the distribution of the diameters of EVs with high RI values from UC, HC, and CRC samples. In both cases, most EVs had diameters in the range of 390 to $420 \mathrm{~nm}$, and there were no significant differences between the examined samples. A similar trend was observed for EVs with lower RI values, which showed smaller morphometric parameters (usually 200 to $325 \mathrm{~nm}$ in diameter), but a greater number of vesicles was found in the CRC samples than in the HC samples.

\section{Ultracentrifugation Affects the Number of EVs with Lower RI Values}

For the HC and CRC samples prepared with the DU isolation method, there were no EVs with lower RI values (Figure 5D). This may depend on the different types of tissue and the procedure itself, as EVs with lower RI values and lower densities may have been damaged by spin speeds that were too high $(180,000 \times g)$. On the other hand, EVs with higher RI values exhibited the same trend for the diameter distribution as those prepared with $\mathrm{U}$ procedure, but there was a higher number of smaller EVs in CRC samples than in the HC samples. These findings suggest that the U procedure did not generate samples with a comparable number and distribution of EVs, and the differentiation of samples was limited and affected by the isolation method.

Tables 4 and 5 show the percentage distribution of EV diameters in UC/HC and CRC samples prepared with the various procedures. Exosomal pellets precipitated from human plasma microsamples using ExoQuick and TEIK reagents contained a higher percentage of the smallest EVs from CRC samples regardless of the RI values, whereas for UC samples, most EVs had diameters from 500 to $1000 \mathrm{~nm}$ 
Table 6 The Ranges of Dry Mass Values of the Highest Percentage of EVs Extracted from Archival Solid Tissue Samples with Different Tumor Grades (G1 to G3) and Proliferative Activities

\begin{tabular}{|c|c|c|c|c|}
\hline Variable & Tumor grade & EV RI class & Dry mass, fg & EVs, $\%$ \\
\hline \multicolumn{5}{|c|}{ Histopathologic diagnosis and tumor grade } \\
\hline \multirow[t]{5}{*}{ Carcinoma } & G1 & High RI & $2.18-6.16$ & 67.72 \\
\hline & & Low RI & $0.75-3.76$ & 90.75 \\
\hline & G2 & High RI & $2.79-78.7$ & 76.23 \\
\hline & & & $21.87-87.46$ & 10.54 \\
\hline & & Low RI & $1.28-3.61$ & 92.67 \\
\hline \multirow[t]{4}{*}{ Adenocarcinoma } & G1 & High RI & $3.17-8.97$ & 69.43 \\
\hline & & & $24.92-99.68$ & 11.80 \\
\hline & & Low RI & $1.15-3.25$ & 80.41 \\
\hline & G3 & High RI & $4.4-12.43$ & 74.95 \\
\hline \multicolumn{5}{|l|}{ Ki-67 nuclear expression } \\
\hline \multirow{9}{*}{ Proliferative activity } & & & $11.518-25.915$ & 26.26 \\
\hline & & & $71.988-287.955$ & 11.99 \\
\hline & & Low RI & $1.082-4.329$ & 51.16 \\
\hline & & & $4.328-9.739$ & 25.52 \\
\hline & High & High RI & $3.818-15.272$ & 55.57 \\
\hline & & & $15.272-34.361$ & 19.39 \\
\hline & & & $95.448-381.792$ & 10.47 \\
\hline & & Low RI & $1.129-4.519$ & 83.93 \\
\hline & & & $4.519-10.168$ & 11.48 \\
\hline
\end{tabular}

EV, extracellular vesicle; G, grade; RI, refractive index.

(ExoQuick), and for TEIK, EVs in the high RI subpopulation were smaller than those in the low RI subpopulation. The obtained results showed that the examination of single EVs prepared using ExoQuick reagent with the DHT method can be used to differentiate of UC/CRC plasma microsamples.

In contrast, a different conclusion can be drawn for the samples obtained with the $U$ and DU procedures. The differentiation of $\mathrm{CRC} / \mathrm{HC}$ samples could only be achieved on the basis of EVs from the high RI fraction with the DU procedure. However, in this case, the high centrifuge speed $(180,000 \times g)$ significantly limits the number of vesicles, which leads to the complete absence of EVs with a low RI.

\section{RI Value of Single EVs Increases with Tumor Grade}

EVs precipitated directly from solid tissue samples of primary CRC were used to investigate the EV heterogeneity based on their RI data and their association with histopathologic diagnosis and tumor grade (Figure 6A). Additional assessments of cell proliferation were performed using the immunohistochemical expression data on the percentage of $\mathrm{Ki}-67-$ positive nuclei among cancer cells. The immunoreactivity of the histological slides was additionally assessed for the coexpression of cytokeratin 20
(CK20), CD63 and Ki-67 using triple stain IHC (Figure 6 F, G, H, I and J). By using the DHT technique, it was possible to digitally stain individual EVs (Figure 6B) using predefined ranges of RI values and to further analyze their optical heterogeneity. Again, it was possible to distinguish two unique subclasses of EVs with higher and lower RI values (Figure 6C). The results showed that for the high RI fraction of EVs, the RI value increased with the tumor grade for both the carcinoma and adenocarcinoma cohorts, but the increase was more obvious for colorectal carcinomas (Figure 6C).

A similar trend was also present for EVs from the low RI fraction, although the extent of change in RI values was less clear. The change in RI values could be explained by a switch in the chemical composition of the EV cargo content, which was confirmed by the determined values of average dry mass density (Figure 6D). The dry mass density of EVs increased and the particles became denser with decreasing degree of differentiation. This effect was much more apparent for EVs from the high RI than those from lower RI fraction. Figure $6 \mathrm{E}$ shows the distribution of the average radius of EVs for the subclasses with higher and lower RI values obtained by fitting the probability density function to histograms of the vesicle radius (Supplemental Figures S3 and S4). For carcinomas, as the degree of the malignancy 

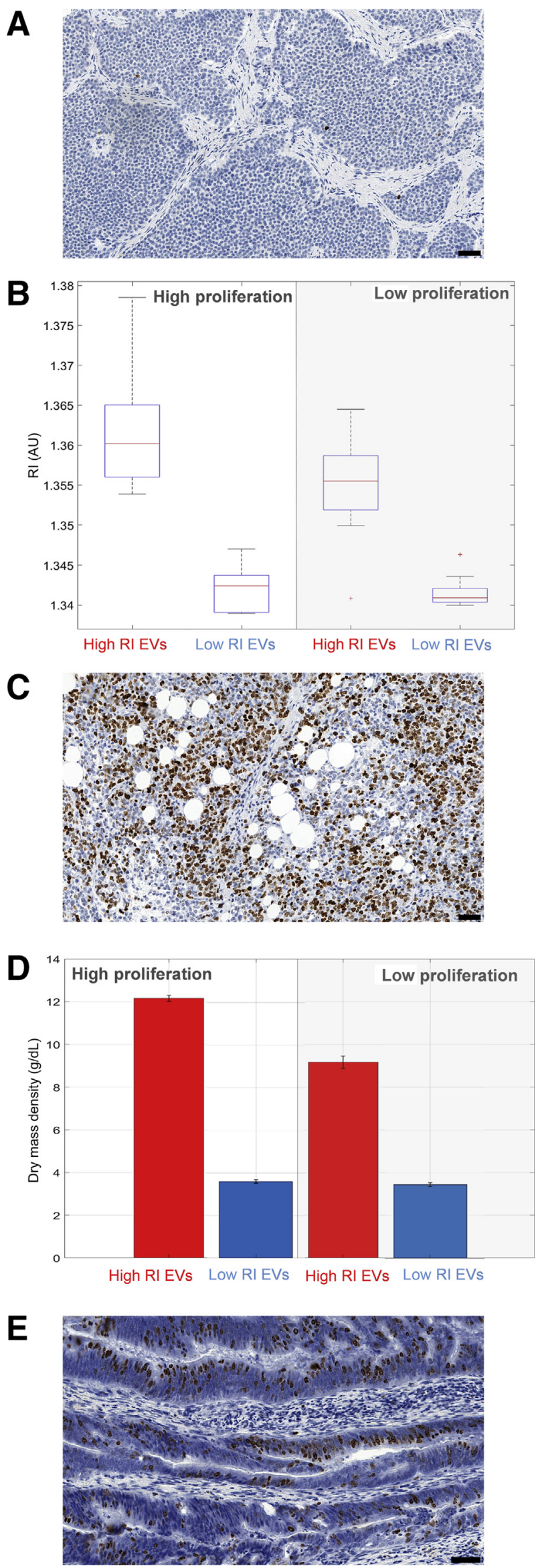

increased, the radius of vesicles with higher RI values increased, whereas for fractions with a low RI, the opposite trend was observed, especially for higher-grade tumors (grade 3 stage). For adenocarcinomas, there was no significant change in the radius values for EVs with a high RI with an increase in the degree of malignancy. However, for EVs with a lower RI, the radius decreased as the tumor grade increased.

After determining the EV size distribution, the dry mass values of the highest percentage of EVs present in the samples were determined. The results (Table 6) showed that the increase in the ranges of dry mass was mainly related to the high RI fraction of EVs. Moreover, the increase in dry mass was correlated with the increase in the degree of malignancy for both carcinomas and adenocarcinomas. These results confirmed a corelation between differences in dry mass and changes in the chemical composition of the EV molecular content, which was denser in cancers with greater malignancy.

\section{Cell Proliferation of CRC Affects the RI of Cancer- Associated EVs}

The examined cancers exhibited different proliferative activities (Figure 7, A, C and E); hence, additional analysis was performed to analyze the relationship of the RI data and EV properties with cell proliferation (Figure 7). The proliferation rate of cancer cells affected the properties of EVs. Increased tumor cell proliferation led to an increase in the average RI value of cancer-associated EVs (Figure 7B). The most significant increase in the RI was observed for the high RI EV fraction. Moreover, increased proliferation caused an increase in dry mass density, which was observed for both subtypes (high/low RI) of EVs, but the most significant differences were noted for high RI EVs (Figure 7D). On the basis of the determined size distribution of vesicles, it was possible to directly determine dry mass values of their cargo for the highest percentage of imaged EVs. The results (Table 6) indicate that greater cancer cell proliferation led to an increase in the percentage of EVs with denser cargo content.

Figure 7 A, C, and E: Micrographs showing immunohistochemical staining against $\mathrm{Ki}-67$ performed on tissue microarray cores with a different tissue expression: $<1 \%$ of positive nuclei $(\mathbf{A}), 71.2 \%$ of positive nuclei $(\mathbf{C})$, and $43.1 \%$ of positive nuclei (E). On the basis of Ki-67 expression level, the tumors were defined as low proliferative lesions, if the percentage of positive nuclei in cancer was $<50 \%$ ( $\mathbf{A}$ and $\mathbf{E})$; the cases exceeding that range were grouped into cancers of high proliferative activity (C). EVs were isolated from frozen solid tissues corresponding to assessed paraffin blocks. B and D: The variation in the average refractive index (RI) values (B) and dry mass density (D) of the high/low RI fraction of extracellular vesicles (EVs) precipitated from solid tissues with different cell proliferation levels. In colorectal tumors with higher cell proliferation, dry mass density increased for high RI EVs, whereas for low RI EVs, it remained at a comparable value regardless of the proliferative activity of cancer cells (D). Scale bars $=50 \mu \mathrm{m}(\mathbf{A}, \mathbf{C}$, and $\mathbf{E})$. AU, arbitrary unit. 

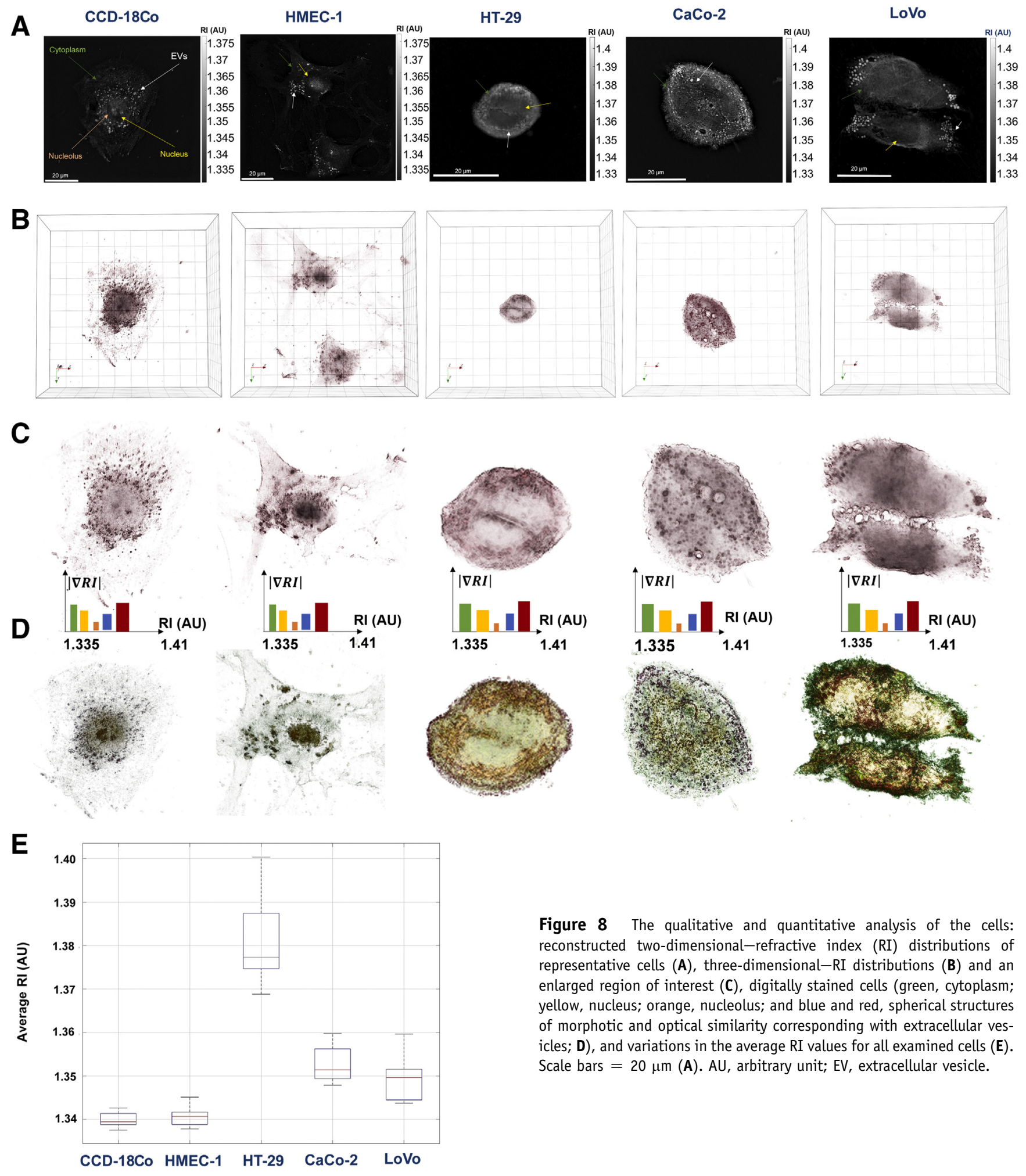

RI Values Differ between EVs and Cells of Their Origin

The 3D spatial distributions of the RI were used to characterize five cell lines: control cells (CCD-18o and HMEC-1) and tumor cells with different grading (HT-29/ grade 1, CaCo-2/grade 2, and LoVo/grade 4). The 2D-RI
Figure 8 The qualitative and quantitative analysis of the cells: reconstructed two-dimensional-refractive index (RI) distributions of representative cells $(\mathbf{A})$, three-dimensional-RI distributions (B) and an enlarged region of interest (C), digitally stained cells (green, cytoplasm; yellow, nucleus; orange, nucleolus; and blue and red, spherical structures of morphotic and optical similarity corresponding with extracellular vesicles; D), and variations in the average RI values for all examined cells (E). Scale bars $=20 \mu \mathrm{m}(\mathbf{A})$. AU, arbitrary unit; EV, extracellular vesicle.

distributions obtained in the plane with the best focus showed that differences between the examined cells could be visualized (Figure 8A). The 2D-RI distributions allow the area occupied by the examined cells to be distinguished and morphologic and structural features to be analyzed. For noncancerous cells, it was possible to distinguish the 

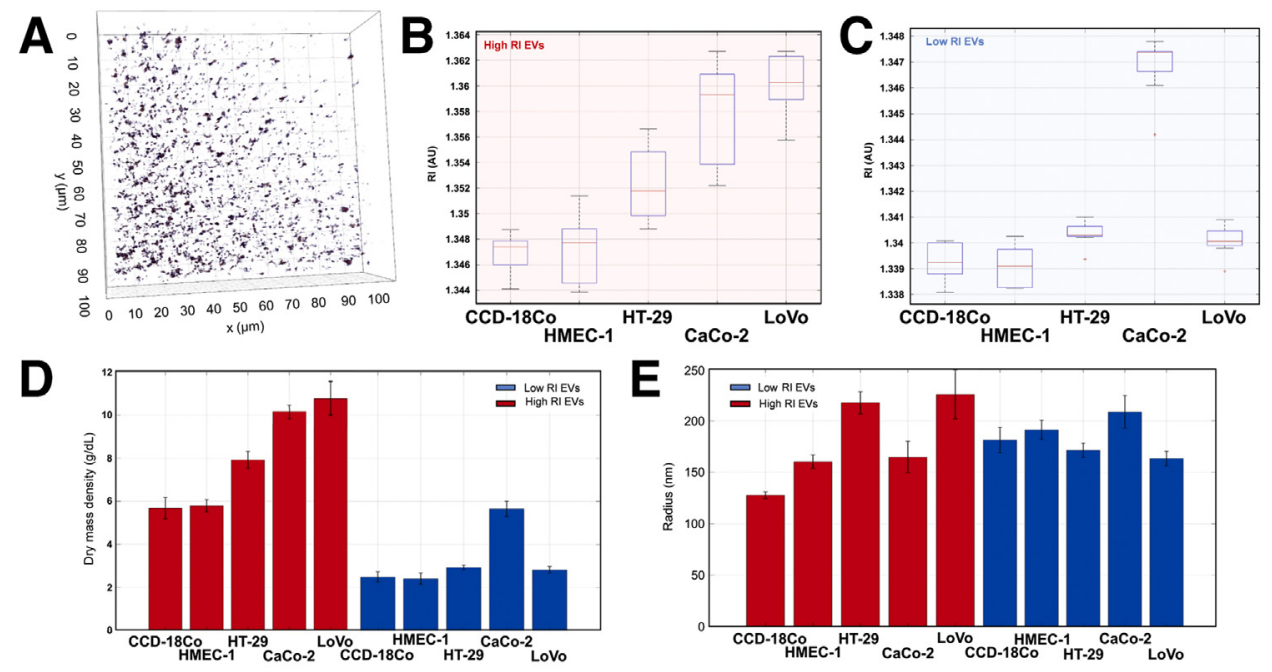

$\mathbf{F}$

PanCK

CD63

Merged
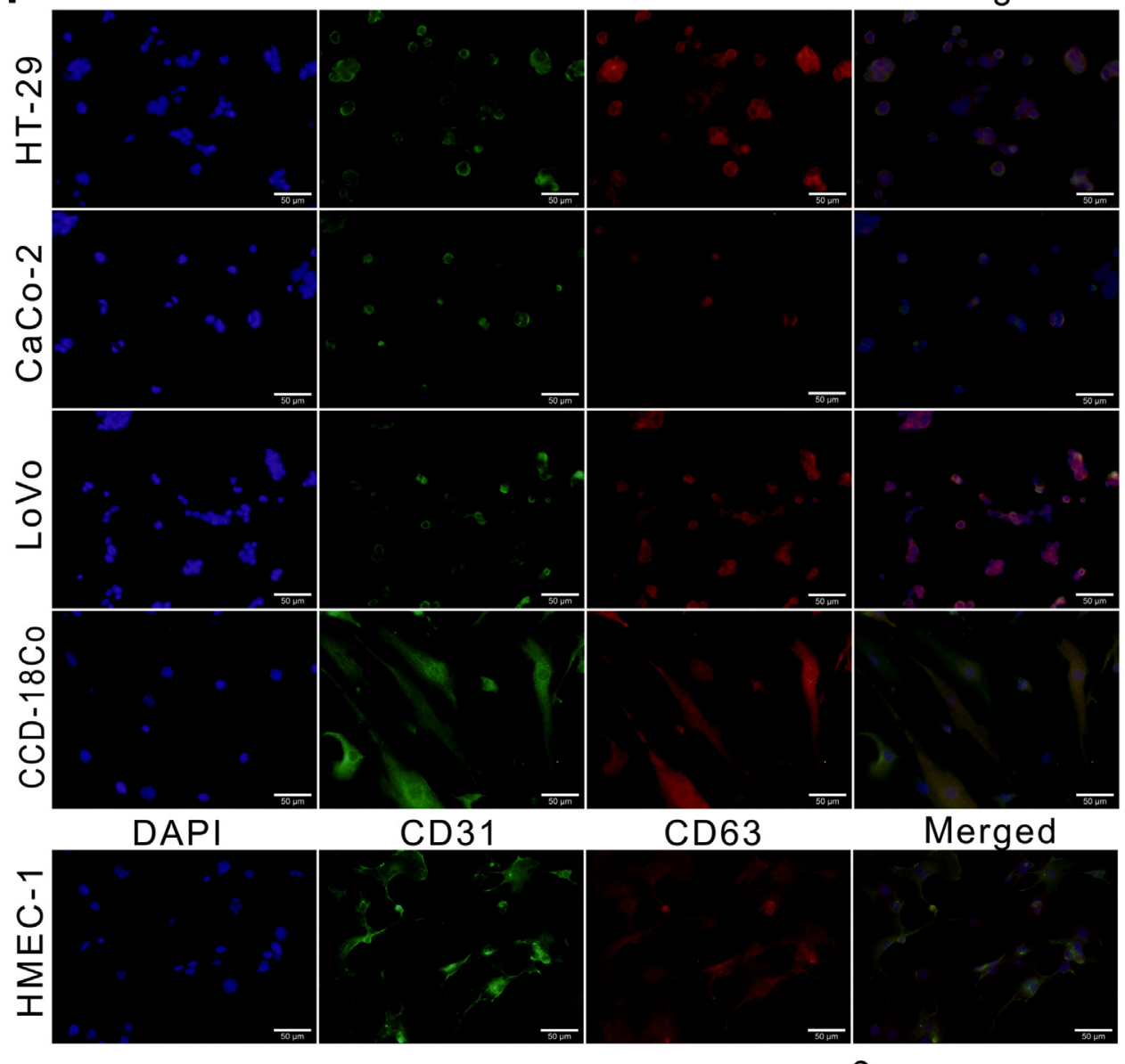

G

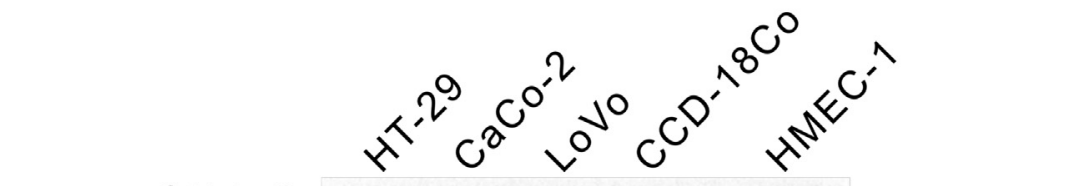

$\beta$-Tubulin (50 kDa)

CD63 (cell pellet, $53 \mathrm{kDa})$ CD63 (EVs from media, $53 \mathrm{kDa}$ ) 
cytoplasm, nucleus, nucleoli, and intracellular spherical structures corresponding to EVs by examining the RI-based contrast. For some tumor cells (especially HT-29 cells), it was difficult to distinguish the nucleus and nucleoli due to the relatively small size of these cells, which led to a large accumulation of intracellular organelles and consequently limited the cell contrast provided by variations in RI values. However, it was still possible to identify intracellular vesicles, which exhibited significantly higher RI values than the other structural elements in the cellular compartment. From the reconstructed RI data, it was possible to obtain high resolution-rendered 3D-RI distributions of cells based on their spatial geometry (Figure 8B) and internal structure. First, to determine the average RI value of whole cells, the whole cellular compartment of individual cells was digitally stained (Figure 8C). Next, on the basis of the variation in the RI values and provided RI-based contrast of the internal cellular microstructures, it was possible to perform labelfree digital staining of the cells (Figure 8D), allowing the visualization of not only the cell area itself but also the cytoplasm, vesicular structures, nuclei, and nucleoli. Full digital staining of the entire cell volume allows the direct analysis of the average RI of the whole cell compartment (Figure 8E). For control cell lines, the average RIs were similar, whereas for cancer cells, they remained higher. One-way analysis of variance showed significant differences between the average RI of healthy and cancer cells $\left(\right.$ Prob $\left.>\mathrm{F}=1.208 \times 10^{-7}\right)$. Moreover, there were significant differences in the average RI among all examined cancer cell lines $\left(\mathrm{Prob}>\mathrm{F}=1.5223 \times 10^{-8}\right)$. The highest average RI value and the highest variance in RI were found in HT-29 cells, which were related to the much smaller size of these cells in comparison to other cells $(\mathrm{CaCo}-2$ and LoVo). The correspondingly higher concentration of cellular content directly led to an increased RI value in these cells. These results indicate that the mean RI value decreases as malignancy increases.

EVs extracted from cancer cell lines with different degrees of malignancy (HT-29, CaCo-2, and LoVo) from both EV subclasses (high/low RI) showed higher RI values (Figure 9A) than EVs from control cell culture samples (CCD-18o and HMEC-1). The greatest differences were found for EVs with higher RI (Figure 9B), where an increasing trend for individual RI values with increasing tumor grade was observed. For EVs in the low RI fraction (Figure 9C), this phenomenon was less pronounced and mainly restricted to EVs extracted from the HT-29 and CaCo- 2 cell lines. For EVs isolated from the LoVo cell line, RI values were higher than for EVs extracted from healthy cells, but those values differed little. Nevertheless, the results of analysis of variance showed that there were significant differences between EVs extracted from normal and cancer cells, although the obtained $P$ values confirmed that greater differences were observed for the high RI fraction of EVs $\left(\right.$ Prob $>$ F: $1.6063 \times 10^{-20}$ ) than the low RI fraction (Prob>F: $6.18034 \times 10^{-12}$ ). The observed changes in the RI value were directly related to the concentration of different components of the EV cargo content, which was correlated with the EV dry mass density. The analysis of RI data showed that for this parameter, a similar trend was observed as for RI value variation (Figure 9D). The high RI fraction had denser cargo content than the low RI fraction of EVs. This may indicate that there are significant differences in the chemical composition of EV cargo related to the degree of tumor cell malignancy.

On the basis of the digitally stained 3D-RI distributions of EVs, it was possible to identify individual EVs and determine their size. The histograms of the EV radius distribution and fitted probability density function are shown in Supplemental Figure S5. The average radii of the EVs from both RI fractions are presented (Figure 9E). For EVs with denser cargo contents, the radius of EVs from HT-29 and LoVo cell lines was higher than that for the control samples (CCD-18Co and HMEC-1), and also that for LoVo cells, for which the highest variation in the EV radius was observed. The high RI EV subtype detected from HT-29 cells had a larger radius than EVs released from CCD-18Co cells, and a similar radius to EVs from HMEC-1 cells respective to the estimated SD. However, for the low RI subtype of EVs, the radius of cancer-associated EVs was higher than that of EVs from the normal control only for the $\mathrm{CaCo}-2$ cell line, whereas its value was lower for EVs from HT-29 and LoVo cells. The radius of EVs from solid tissue CRC samples was significantly larger than that of EVs detected from cell lines.

After determining the EV size distribution, it was possible to determine the dry mass of particular EVs from the dry mass density evaluated on the basis of RI data. The obtained results are shown in Table 7, where the dry mass ranges for the most representative percentage of EVs in the samples were determined. For EVs released from cancer cells, the dry mass of the

\footnotetext{
Figure 9 The refractive index (RI) of extracellular vesicles (EVs) increased in cancer cell lines (HT-29, CaCo-2, and LoVo) and with their corresponding tumor grade (G; G1, G2, and G4, respectively). A-E: RI data-based characterization of the heterogeneity of EVs extracted from cancer cells: representative three-dimensional-RI distribution of EVs extracted from CaCo-2 cells (A), variations in the RI values for the high RI EV subclass (B), variations in the RI values for low RI EVs (C), average dry mass density (D), and average radius of vesicles from each (low/high RI) EV fraction (E). Cell lines defined as normal control cultured of epithelial colonic cells (CCD-18Co) and endothelial cells (HMEC-1) showed EVs of decreased RI value. F: A fluorescent staining against pancytokeratin, AE1/AE3 clone (PanCK), was used to label all epithelial cells. HMEC-1 cells were contrary stained with a CD31 antibody to demonstrate an endothelial origin. In each case, CD63 tetraspanin was used as cellular target for confirmation of the exosomal nature of examined cell lines. F: Individual cells showed varied fluorescence intensity against CD63 staining, which is presented in attached micrographs. The observed differences could also be recognized at the protein level for individual cell pellets. G: As assumed, EVs expressed a different protein level of tetraspanin compared with the cell cultures from which they were harvested. Scale bars $=50 \mu \mathrm{m}(\mathbf{F})$. AU, arbitrary unit.
} 
Table 7 The Ranges of Dry Mass Values of the Highest Percentage of EVs Precipitated from Cell Lines

\begin{tabular}{lllll}
\hline Variable & Cell line & EV RI class & Dry mass, fg & EVs, \% \\
\hline Controls & CCD-18Co & High RI & $1.78-7.13$ & 94.94 \\
& & Low RI & $0.78-3.13$ & 91.62 \\
& HMEC-1 & High RI & $1.82-16.34$ & 85.33 \\
& & Low RI & $0.76-6.83$ & 85.33 \\
G1 & HT-29 & High RI & $2.49-22.37$ & 80.00 \\
& & Low RI & $0.92-8.3$ & 89.41 \\
G2 & \multirow{2}{*}{ CaCo-2 } & High RI & $3.22-28.94$ & 89.14 \\
& \multirow{3}{*}{ G4 } & Low RI & $1.77-15.97$ & 92.81 \\
& LoVo & High RI & $3.52-31.64$ & 83.56 \\
& & Low RI & $0.89-7.99$ & 94.24 \\
\hline
\end{tabular}

EV, extracellular vesicle; G, grade; RI, refractive index.

fractions with high/low RIs was higher than for that of EVs from healthy controls and, in general, that increase was mainly related to EVs with high RI values. Moreover, the dry mass value increased with the increase in the degree of malignancy, which suggests that significant differences in the chemical composition of the EV cargo are associated with CRC progression.

\section{Long-Term Storage of Samples at Deep-Freeze Temperature May Affect the Levels of Proteins Contained in EVs}

In this study, EVs were isolated from both freshly collected and archival samples stored at deep-freezing temperatures. Although it was possible to effectively isolate EVs from both sample types, the levels of the investigated biomarkers that are more frequently associated with the EVs may have varied with respect to sample storage conditions. A good example was the protein level of CD63 tetraspanin, which is one of the most frequently examined target proteins for the confirmation of the exosomal nature of sample and, hence, was performed in this study. According to immunoblotting analyses performed on archived (Figure 3C) and freshly obtained cell pellets precipitated from HT-29 cells (Figure 9G), the protein level of CD63 significantly differed between the samples; however, in both cases, cells were cultured in comparable conditions. An archival cell pellet from 2018 was negative for CD63, but the pellet obtained from fresh cells was confirmed to have the positive bands for this tetraspanin. CD63 positivity was also confirmed in freshly cultured HT-29 cells with immunofluorescence (Figure 9F). For EV isolates obtained from archival solid tissues, control reactions against CD63 were performed on the corresponding formalin-fixed, paraffin-embedded blocks that were confirmed to be positive and, in most cases, pronounced expression of this tetraspanin was observed (Figure 6, $\mathrm{F}-\mathrm{I}$ ). Control immunoblotting reactions were also performed for EVs isolated from archival solid tissues, but the bands were poorly represented compared with those in EV fractions precipitated from fresh plasma microsamples (Supplemental Figure S6).

\section{Discussion}

To date, only one technique based on NTA has been used to characterize the average RIs of EVs. It indicated differences in the heterogeneity of individual EVs in relation to their diameter, where large vesicles showed higher RI values than small EVs, ${ }^{25}$ and the estimated RI values were accurate to three decimal places. Nevertheless, the high heterogeneity of EVs makes it difficult to measure vesicle ultrastructure with the NTA method because of technical parameters, which include camera-level and detection threshold variables. ${ }^{43}$ Other limitations of NTA include the need to dilute the examined samples and the limited ability to phenotype EVs based on the detection of their fluorescence, which also requires the use of specific dyes or directly labeled appropriate monoclonal antibodies. For quantitative measurements, this method does not always allow for a reliable assessment of EVs, which are positive for the examined biomarkers. ${ }^{44}$ The NTA method is also a costly, time-consuming procedure that requires the involvement of specialized technical staff. Moreover, the RI measured by the NTA method does not allow for the assessment of the spatial heterogeneity of individual vesicles, especially if there is a need to examine changes in their 3D structure. This approach used an indirect size estimation method to determine the size dependency RI of the EVs and assumed that examined objects with a similar size behaved similarly. However, possible differences in the scattering of single homogeneous spheres versus multilayered spheres (double layer) or the different and unknown contents of the cargo of EVs with different sizes were not considered.

The present study showed that DHT can more precisely examine EV heterogeneity. It is possible to visualize the $\mathrm{EVs}$ in real time, and the sample preparation does not require the prior use of exogenous labeling agents or dyes that could affect the primary physicochemical parameters of the vesicles. The 3D-RI tomograms provide direct information about the morphology and spatial distribution of their RI with a higher resolution than NTA. Digital staining of EVs enables the volumetric characterization of the heterogeneity of their RI distribution with submicrometer or even subnanometer resolution below the limitation of classical optical microscopy techniques. The high diversity of the molecular content of EVs emphasizes their complexed functionality, as these vesicles have the potential to transport both coding and noncoding DNA and RNA particles, lipids, and numerous proteins. ${ }^{45}$ Therefore, the 3D-RI tomograms related to the local changes in the density can also be dependent on the changes in EV content. The study showed that DHT can be used to differentiate EVs based on their spatial RI distribution, but there is no clear association 
or dependence between the EV RI values and their size, as stated regarding the results of NTA analysis. DHT analysis indicated that EVs with the same 3D-RI distribution can have different diameters. These measurements are limited to only a few samples of examined pellets. However, such analyses may confirm the clinical value of differentiating individual pellet samples from patients with different diagnoses. IEVs isolated from normal colonic mucosa and UC had significantly larger diameters than those isolated from CRC. This observation may suggest the existence of different EV subpopulations in cancer, inflamed colon, and healthy colonic mucosa. Moreover, these measurements show that the EV subpopulation is extremely diverse even within the same sample and that vesicles with similar morphologic parameters may present significant differences. EDS maps and spectral analyses of exosomal pellets from human plasma confirmed that there were significant differences in chemical elements and compounds at the level of a single vesicle based on the medical diagnosis.

Depending on the sample preparation procedure used, the retrieved 3D-RI tomograms of EVs exhibited significant differences. Moreover, different procedures also affected the number of EVs that could be extracted from 3D-RI tomograms, which could have led to the different size distributions of EVs in samples. This phenomenon was observed for TEIK, in which the smaller EVs with higher RI values aggregated and merged to form clusters, as well as for the DU isolation procedure, in which the EVs of lower RI values were not present. Interestingly, in ultracentrifuged samples, lEVs with large diameters were present despite high centrifugation speeds. The lEVs with significantly larger diameters have been observed in UC samples in particular. For the ultracentrifugation procedure, most of EV population with diameters $<200 \mathrm{~nm}$ could not be identified in those pelleted samples, which could be related to the limited resolution of DHT. Nevertheless, IEVs have been identified in UC samples with larger diameters than those in CRC in EV pellets precipitated using commercial reagents, which may confirm the existence of qualitative differences that depend on the type of tissue examined. In this study, ultracentrifugation significantly reduced the number of EVs, especially in relation to $\mathrm{lEVs}$ with diameters corresponding to microvesicles and oncosomes. This calls into question the use of $U$ and DU methods for the isolation of EVs to verify the importance of extracellular communication in the pathogenesis of the studied diseases, which causes the loss of a significant population of larger EVs with potential pathogenetic importance. Commercially used reagents were also shown to potentially have a different impact on the RI value of the observed vesicles, which may be due to the different effect of the reagent on the suspended EV fractions. The sample preparation procedure was previously reported to influence RI data during the fixation of eukaryotic cells, ${ }^{36}$ which confirms the observations for the examined EVs. Therefore, during the visualization of EVs with the DHT method, attention should be given to the sample preparation procedure, when using RI data for reliable studies in the life and medical sciences.

In addition to analyzing fresh tissue samples, this study also examined EVs from archival samples. For these analyses, 11 frozen solid tissue samples obtained from patients diagnosed with CRC were selected for further examination. In two cases of CRC with corresponding healthy surgical margins representing normal colonic mucosa, EVs were isolated using two ultracentrifugation procedures. The optical and morphometric parameters of single EVs of the samples were compared using DHT. A higher speed of centrifugation affected the number of isolated EVs. Moreover, there were significant differences between EVs isolated using different ultracentrifugation approaches. In the remaining nine archival CRC samples, EVs were isolated from solid tissues using commercially available reagents (the ExoQuick method). DHT imaging of those EVs showed that the vesicles with higher RI values accurately reflected the histopathologic patterns of CRC. The subtype of EVs defined for the first time as high RI-EVs showed a positive association with the tumor grade and proliferative activity of CRC cells. Moreover, the content of high RI EVs showed a denser dry mass, which was also dependent on the tumor grade. Considering, among the selected CRC cases, grade 3 had higher nuclear expression of the Ki-67 antigen, it was difficult to determine which histopathologic parameter had a more pronounced impact on the observed differences in the distribution of EVs with higher RI values. Thus, an additional comparison of the optical properties of EVs was performed in selected in vitro models. For this analysis, EVs were isolated from the fresh culture media of selected cell lines representing CRC with different tumor grades with increasing levels of malignancy, including grade stage (HT29, CaCo-2, and LoVo) and from cell lines that were defined as controls of epithelial (CCD-18Co) and endothelial origins (HMEC-1). Consistent with the results of the examination of archival samples, the cell line models confirmed that the dry mass density of cancer-associated EVs and their RI values increased with the grade and malignancy of the studied cancer cells, as higher values were observed for those parameters in metastatic case of CRC (LoVo cells). In the case of controls (CCD-18Co and HMEC-1 cells), the isolated EVs from those cells had lower levels of both studied hallmarks than cancer cells. This conclusion may suggest that molecular changes occur in EV cargo during carcinogenesis in CRC as well as for lesions of different biological malignancy.

In summary, this study supports the use of DHT as a suitable method for the label-free imaging of EVs at the single vesicle level. In this article, a new classification of EVs was also proposed according to noted differences in selected optical parameters. These new subtypes of EVs were based on the RI value of individual vesicles. Interestingly, it has been hypothesized that EVs with higher RI values, entitled as high RI-EVs, may support routine histopathologic diagnosis and early screening of CRC, because 
they accurately reflect the changes that occur in the tumor microenvironment. Nevertheless, the proposed division of EVs into two different vesicle subclasses also has limitations. First, the obtained RI values for EVs with low/high RI depended on the tissue type and the EV isolation method, which means that further research should be performed to establish optimal RI ranges for these two EV classes, considering different conditions of sample precipitations and origin. Second, additional molecular phenotyping of these EVs should be performed to establish a relationship between RI and cargo content and the corresponding vesicle subtype. However, such analyses face technological limitations related to the need to initially separate the appropriate EVs into those with high and low RI before their molecular phenotyping.

\section{Acknowledgments}

We thank the patients who participated in this study; Dr. Krzysztof Rogala for assistance in recruiting patients and collecting tissue material from patients undergoing endoscopic diagnostics; Dr. Mariusz Chabowski for providing tissue samples taken from colectomy procedures; and 4th Military Hospital (Wroclaw, Poland) for consenting to this study.

\section{Author Contributions}

Ł.Z. developed methods and performed conceptualization, investigation, formal analysis, resources, data curation, visualization, validation, writing (original draft, review, and editing), and project administration, and acquired funding and resources; I.B. developed methods and performed conceptualization, investigation, formal analysis, data curation, visualization, validation, software, and writing (original draft, review, and editing); A.U.J. developed methods and performed investigation, formal analysis, and writing (original draft); A.R. developed methods and performed investigation, formal analysis, and writing (original draft); M.K. developed methods and performed formal analysis; I.C. performed investigation, formal analysis, and writing (review and editing); P.D. performed supervision and writing (review and editing).

\section{Supplemental Data}

Supplemental material for this article can be found at http://doi.org/10.1016/j.ajpath.2021.08.005.

\section{References}

1. Perdomo L, Vidal-Gómez X, Soleti R, Vergori L, Duluc L, Chwastyniak M, Bisserier M, Le Lay S, Villard A, Simard G, Meilhac O, Lezoualc'h F, Khantalin I, Veerapen R, Dubois S, Boursier J, Henni S, Gagnadoux F, Pinet F, Andriantsitohaina R,
Martínez MC; Metabol Study Group: Large extracellular vesicleassociated Rap1 accumulates in atherosclerotic plaques, correlates with vascular risks and is involved in atherosclerosis. Circ Res 2020, 127:747-760

2. Rak J: Cancer: organ-seeking vesicles. Nature 2015, 527:312-314

3. Ciardiello C, Leone A, Lanuti P, Roca MS, Moccia T, Minciacchi VR, Minopoli M, Gigantino V, De Cecio R, Rippa M, Petti L, Capone F, Vitagliano C, Milone MR, Pucci B, Lombardi R, Iannelli F, Di Gennaro E, Bruzzese F, Marchisio M, Carriero MV, Di Vizio D, Budillon A: Large oncosomes overexpressing integrin alpha-V promote prostate cancer adhesion and invasion via AKT activation. J Exp Clin Cancer Res 2019, 38:317

4. Gu TW, Wang MZ, Niu J, Chu Y, Guo KR, Peng LH: Outer membrane vesicles derived from E. coli as novel vehicles for transdermal and tumor targeting delivery. Nanoscale 2020, 12:18965-18977

5. Dou G, Tian R, Liu X, Yuan P, Ye Q, Liu J, Liu S, Zhou J, Deng Z, Chen X, Liu S, Jin Y: Chimeric apoptotic bodies functionalized with natural membrane and modular delivery system for inflammation modulation. Sci Adv 2020, 6:eaba2987

6. Ma Z, Wang Y, Li H: Applications of extracellular vesicles in tissue regeneration. Biomicrofluidics 2020, 14:011501

7. Popowski K, Lutz H, Hu S, George A, Dinh PU, Cheng K: Exosome therapeutics for lung regenerative medicine. J Extracell Vesicles 2020, 9:1785161

8. Ciardiello C, Migliorino R, Leone A, Budillon A: Large extracellular vesicles: size matters in tumor progression. Cytokine Growth Factor Rev 2020, 51:69-74

9. Luo P, Mao K, Xu J, Wu F, Wang X, Wang S, Zhou M, Duan L, Tan Q, Ma G, Yang G, Du R, Huang H, Huang Q, Li Y, Guo M, Jin Y: Metabolic characteristics of large and small extracellular vesicles from pleural effusion reveal biomarker candidates for the diagnosis of tuberculosis and malignancy. J Extracell Vesicles 2020, 9:1790158

10. Witwer KW, Buzás EI, Bemis LT, Bora A, Lässer C, Lötvall J, Nolte-'t Hoen EN, Piper MG, Sivaraman S, Skog J, Théry C, Wauben MH, Hochberg F: Standardization of sample collection, isolation and analysis methods in extracellular vesicle research. J Extracell Vesicles 2013, 2. doi: $10.3402 /$ jev.v2i0.20360

11. Negahdaripour M, Owji H, Eskandari S, Zamani M, Vakili B, Nezafat N: Small extracellular vesicles (sEVs): discovery, functions, applications, detection methods and various engineered forms. Expert Opin Biol Ther 2020, 21:371-394

12. Pezzicoli G, Tucci M, Lovero D, Silvestris F, Porta C, Mannavola F: Large extracellular vesicles-a new frontier of liquid biopsy in oncology. Int J Mol Sci 2020, 21:6543

13. van der Pol E, Coumans FA, Grootemaat AE, Gardiner C, Sargent IL, Harrison P, Sturk A, van Leeuwen TG, Nieuwland R: Particle size distribution of exosomes and microvesicles determined by transmission electron microscopy, flow cytometry, nanoparticle tracking analysis, and resistive pulse sensing. J Thromb Haemost 2014, 12:1182-1192

14. Panagopoulou MS, Wark AW, Birch DJS, Gregory CD: Phenotypic analysis of extracellular vesicles: a review on the applications of fluorescence. J Extracell Vesicles 2020, 9:1710020

15. Kuś A, Krauze W, Kujawińska M: Limited-angle holographic tomography with optically controlled projection generation. Edited by Brown TG, Cogswell CJ, Wilson T. In Three-Dimensional and Multidimensional Microscopy: Image Acquisition and Processing XXII. Bellingham, WA: SPIE, 2015. pp. 933007

16. Oh J, Ryu JS, Lee M, Jung J, Han S, Chung HJ, Park Y: Threedimensional label-free observation of individual bacteria upon antibiotic treatment using optical diffraction tomography. Biomed Opt Express 2020, 11:1257

17. Kuś A, Dudek M, Kemper B, Kujawińska M, Vollmer A: Tomographic phase microscopy of living three-dimensional cell cultures. J Biomed Opt 2014, 19:046009

18. Lauer $\mathrm{V}$ : New approach to optical diffraction tomography yielding a vector equation of diffraction tomography and a novel tomographic microscope. J Microsc 2002, 205:165-176 
19. Kim K, Yoon J, Shin S, Lee S, Yang SA, Park Y: Optical diffraction tomography techniques for the study of cell pathophysiology. J Biomed Photon Eng 2016, 2. 020201-1-020201-16

20. Jung JH, Hong SJ, Kim HB, Kim G, Lee M, Shin MS, Lee S, Kim DJ, Lee CG, Park Y: Label-free non-invasive quantitative measurement of lipid contents in individual microalgal cells using refractive index tomography. Sci Rep 2018, 8:6524

21. Kim TK, Lee BW, Fujii F, Kim JK, Pack CG: Physicochemical properties of nucleoli in live cells analyzed by label-free optical diffraction tomography. Cells 2019, 8:699

22. Zadka Ł, Chrabaszcz K, Buzalewicz I, Wiercigroch E, GlatzelPlucińska N, Szleszkowski Ł, Gomułkiewicz A, Piotrowska A, Kurnol K, Dzięgiel P, Jurek T, Malek K: Molecular profiling of the intestinal mucosa and immune cells of the colon by multi-parametric histological techniques. Sci Rep 2021, 11:11309

23. Cotte Y, Toy F, Jourdain P, Pavillon N, Boss D, Magistretti P, Marquet P, Depeursinge C: Marker-free phase nanoscopy. Nat Photon 2013, 7:113-117

24. Salucci S, Battistelli M, Burattini S, Sbrana F, Falcieri E: Holotomographic microscopy: a new approach to detect apoptotic cell features. Microsc Res Tech 2020, 83:1464-1470

25. Gardiner C, Shaw M, Hole P, Smith J, Tannetta D, Redman CW, Sargent IL: Measurement of refractive index by nanoparticle tracking analysis reveals heterogeneity in extracellular vesicles. J Extracell Vesicles 2014, 3:25361

26. Harpaz N, Polydorides AD: Colorectal dysplasia in chronic inflammatory bowel disease: pathology, clinical implications, and pathogenesis. Arch Pathol Lab Med 2010, 134:876-895

27. Menter DG, Davis JS, Broom BM, Overman MJ, Morris J, Kopetz S: Back to the colorectal cancer consensus molecular subtype future. Curr Gastroenterol Rep 2019, 21:5-24

28. Zadka Ł, Piotrowska A, Opalińska A, Haczkiewicz-Leśniak K, Grybowski D, Ceremuga I, Chabowski M, Dzięgiel P: Comparative analysis of exosome markers and extracellular vesicles between colorectal cancer and cancer-associated normal colonic mucosa. Pol Arch Intern Med 2020, 130:640-648

29. Zheng X, Xu K, Zhou B, Chen T, Huang Y, Li Q, Wen F, Ge W, Wang J, Yu S, Sun L, Zhu L, Liu W, Gao H, Yue L, Cai X, Zhang Q, Ruan G, Zhu T, Wu Z, Zhu Y, Shao Y, Guo T, Zheng S: A circulating extracellular vesicles-based novel screening tool for colorectal cancer revealed by shotgun and data-independent acquisition mass spectrometry. J Extracell Vesicles 2020, 9:1750202

30. Reggiani Bonetti L, Barresi V, Bettelli S, Domati F, Palmiere C: Poorly differentiated clusters (PDC) in colorectal cancer: what is and ought to be known. Diagn Pathol 2016, 11:31

31. Tong G, Zhang G, Liu J, Zheng Z, Chen Y, Niu P, Xu X: Cutoff of $25 \%$ for Ki67 expression is a good classification tool for prognosis in colorectal cancer in the AJCC-8 stratification. Oncol Rep 2020, 43: $1187-1198$
32. Vella LJ, Scicluna BJ, Cheng L, Bawden EG, Masters CL, Ang CS, Willamson N, McLean C, Barnham KJ, Hill AF: A rigorous method to enrich for exosomes from brain tissue. J Extracell Vesicles 2017, 6: 1348885

33. Théry C, Amigorena S, Raposo G, Clayton A: Isolation and characterization of exosomes from cell culture supernatants and biological fluids. Edited by Bonifacino JS, Green KJ, Lippincott-Schwartz J, Yamada K, Youle R. In Current Protocols in Cell Biology. John Wiley \& Sons, Inc., 2006. pp. 1-29

34. Laemmli UK: Cleavage of structural proteins during the assembly of the head of bacteriophage T4. Nature 1970, 227:680-685

35. Corrêa ACNTF, Patricia R, Pereira PR, Vânia M, Paschoalin VMF F: Preparation and characterization of nanoliposomes for the entrapment of bioactive hydrophilic globular proteins. J Vis Exp 2019, 150: e59900

36. Baczewska M, Eder K, Ketelhut S, Kemper B, Kujawińska M: Refractive index changes of cells and cellular compartments upon paraformaldehyde fixation acquired by tomographic phase microscopy. Cytometry A 2021, 99:388-398

37. Barer R: Interference microscopy and mass determination. Nature 1954, 169:366-367

38. Friebel M, Meinke M: Model function to calculate the refractive index of native hemoglobin in the wavelength range of 250-1100 nm dependent on concentration. Appl Opt 2006, 45:2838-2842

39. Phillips KG, Jacques SL, McCarty OJ: Measurement of single cell refractive index, dry mass, volume, and density using a transillumination microscope. Phys Rev Lett 2012, 109:118105

40. Aknoun S, Savatier J, Bon P, Galland F, Abdeladim L, Wattellier B, Monneret S: Living cell dry mass measurement using quantitative phase imaging with quadriwave lateral shearing interferometry: an accuracy and sensitivity discussion. J Biomed Opt 2015, 20:126009

41. Abràmoff $\mathrm{MD}$, Magalhães $\mathrm{PJ}$, Ram SJ: Image processing with ImageJ. Biophotonics Int 2004, 11:36-42

42. Théry C, Witwer KW, Aikawa E, Alcaraz MJ, Anderson JD, Andriantsitohaina R, et al: Minimal information for studies of extracellular vesicles 2018 (MISEV2018): a position statement of the International Society for Extracellular Vesicles and update of the MISEV2014 guidelines. J Extracell Vesicles 2018, 7:1535750

43. Maas SL, de Vrij J, van der Vlist EJ, Geragousian B, van Bloois L, Mastrobattista E, Schiffelers RM, Wauben MH, Broekman ML, Nolte-'t Hoen EN: Possibilities and limitations of current technologies for quantification of biological extracellular vesicles and synthetic mimics. J Control Release 2015, 200:87-96

44. Szatanek R, Baj-Krzyworzeka M, Zimoch J, Lekka M, Siedlar M, Baran J: The methods of choice for extracellular vesicles (EVs) characterization. Int J Mol Sci 2017, 18:1153

45. Veziroglu EM, Mias GI: Characterizing extracellular vesicles and their diverse RNA contents. Front Genet 2020, 11:700 